\title{
A LUNAR OCCULTATION AND DIRECT IMAGING SURVEY OF MULTIPLICITY IN THE OPHIUCHUS AND TAURUS STAR-FORMING REGIONS ${ }^{1}$

\author{
M. Simon, ${ }^{2}$ A. M. Ghez, ${ }^{3,4}$ Ch. Leinert, ${ }^{5}$ L. Cassar, ${ }^{6}$ W. P. Chen, ${ }^{7}$ R. R. Howell, ${ }^{8}$ R. F. Jameson, ${ }^{8,9}$ \\ K. Matthews, ${ }^{4}$ G. Neugebauer, ${ }^{4}$ AND A. Richichi ${ }^{5}$ \\ Received 1994 May 18; accepted 1994 October 28
}

\begin{abstract}
We present an IR lunar occultation and direct imaging search for companions in the Ophiuchus starforming region and update a similar search of the Taurus region. The search is sensitive to companions in the angular separation range $0.005-10^{\prime \prime}$. In Ophiuchus we surveyed 35 young star targets; this sample contains at least 10 binaries, two triples, and one quadruple. Ten of the companion stars are newly discovered. In Taurus, the survey now includes 47 systems among which there are at least 22 binaries and four triples. Only two companion stars are newly identified because there is strong overlap with prior work. All the triples and quadruple are hierarchical. The observed binary frequency in Ophiuchus, in the 3-1400 AU range of separations, is at least $1.1 \pm 0.3$ that of the nearby solar-like stars. This value is a lower bound because we make no corrections for incompleteness. In Taurus, in the same range of separations, the observed binary frequency is at least $1.6 \pm 0.3$ that of the nearby solar-like stars. This value extends Ghez et al.'s (1993) and Leinert's et al.'s (1993) determination of an excess binary frequency to 3 AU separation. We used the WT/TT type and the $K-L$ color index to distinguish between systems with and without inner disks. We find no convincing difference in the binary frequency or distribution of separations of the systems with and without inner disks. The $1.3 \mathrm{~mm}$ continuum emission of the single systems exceeds that of the multiples suggesting that their extensive outer disks are more massive. The specific angular momenta of the binaries overlap those of molecular cloud cores measured by Goodman et al. (1993).
\end{abstract}

Subject headings: binaries: visual - occultations - open clusters and associations: general stars: pre-main-sequence

\section{INTRODUCTION}

Techniques offering unprecedented angular resolution in the visible and near-IR are revealing a population of young star binaries that until recently had only been suspected. We now know that companionship among the young stars is common. In the angular separation ranges to which recent surveys are sensitive, the binary frequency is greater in the Taurus and Ophiuchus star-forming regions (SFRs) than among the nearby solar-like stars (Ghez, Neugebauer, \& Matthews 1993; Leinert et al. 1993). In the remarkably dense Orion Trapezium cluster, the binary frequency is nearly identical to that of the nearby solar-like stars (Prosser et al. 1994). In addition, visual binaries appear common in southern hemisphere SFRs

\footnotetext{
${ }^{1}$ From our first contact, through the observations of 1991 and a rained-out attempt in 1992, David Allen did his best to make the AAT observations a success. It was characteristic that he asked, in 1994 May, that he not appear as a coauthor. David Allen died on 1994 July 26. We dedicate the paper to his memory.

${ }^{2}$ Astronomy Program, State University of New York, Stony Brook, NY 11794.

${ }^{3}$ Department of Astronomy, University of California, Los Angeles, CA 90024.

${ }^{4}$ Palomar Observatory, California Institute of Technology, Pasadena, CA 91125.

5 Max-Planck-Institut für Astronomie, Königstuhl 17, D-69117 Heidelberg, Germany.

${ }^{6}$ Department of Mathematics and Science, US Merchant Marine Academy, Kings Point, NY 11024.

${ }^{7}$ Institute of Astronomy and Department of Physics, National Central University, 32054 Chung-Li, Taiwan.

${ }^{8}$ Physics and Astronomy Department, University of Wyoming, Box 3905, University Station, Laramie, WY 82071.

${ }^{9}$ Astronomy Group, University of Leicester, University RD., Leicester LE1 7RH, England.
}

(Reipurth \& Zinnecker 1993; Hughes et al. 1994). For astronomers to learn what properties of the molecular cloud birthplace determine the star formation rate, the binary frequency, and the distribution of binary orbital parameters, these surveys need to be extended to include the full range of binary orbits and a more complete sampling of the diverse SFRs. Detailed study of individual binaries will probe the earliest phases of stellar evolution and will enable the measurement of fundamental properties such as their mass and age.

The binary frequency among young stars also bears on the problems of planet formation. The circumstellar disks associated with young stars contain the source material for planets. Tidal interactions in a binary system couple the stars and their circumstellar disks, thereby affecting the subsequent evolution of binary orbits and disk structure. Examples of truncated circumstellar disks and circumbinary disks have been identified (Mathieu, Adams, \& Latham 1991; Simon \& Guilloteau 1992; Dutrey, Guilloteau, \& Simon 1994; Ghez et al. 1994). The implications for planet formation in binary systems must be significant and deserve thorough investigation.

Of the available techniques, lunar occultations can reach the highest angular resolution: in the IR $K$ band $(2.2 \mu \mathrm{m})$ the limiting resolution is $\sim 0$ ".005 (Simon et al. 1992). Although only one-dimensional, occultations offer resolution more than a factor of 10 higher than that presently attainable by speckle imaging at $K$ on $4 \mathrm{~m}$ class telescopes. The Taurus and Ophiuchus SFRs lie in the zodiac so both can be studied by the occultation technique. Moreover, since they are at about the same distance, $\sim 140$ pc (Elias 1978b; de Geus \& Burton 1991), the range of physical separations resolved is approximately the same. The structures of these two SFRs set the pace for occultation observations. In Taurus, star-forming activity 
occurs in clumps distributed over more than $20 \mathrm{pc}$, or $10^{\circ}$ on the sky (e.g., Kenyon et al. 1990, Fig. 1), so occultations of different young stars become observable as the Moon passes over the region. In contrast, star formation in Ophiuchus is concentrated in a very dense core $\sim 1 \times 2$ pc in extent (e.g., Wilking 1989). Occultations of stars in this core are concentrated into about one year and recur at intervals of about 6 and $12 \mathrm{yr}$. The Moon passed across the core of the Ophiuchus SFR in 1991 and 1992. We used this opportunity to observe its young stars by lunar occultation in order to carry out exactly the same kind of survey for multiplicity as is in progress for the Taurus SFR (Simon et al. 1992). The next series of occultations of the core of Ophiuchus will occur in 2003-2005. We obtained occultation data for 35 young star systems and supplemented these data with IR images to cover a larger range of angular separations.

This paper presents the results of our Ophiuchus survey in $\S 2$. The survey of the Taurus SFR now includes 47 systems; we update the results in $\S 3$. We compare the multiplicity properties of the Ophiuchus and Taurus SFRs to each other and to those of the solar-like stars in $\S 4$. We discuss the physical properties of the singles and the multiples, and their circumstellar disks in $\S 5$.

\section{THE OPHIUCHUS MULTIPLICITY SURVEY}

\subsection{The Objects Observed}

Our object list for the Ophiuchus SFR is derived from the works of Elias (1978a), Wilking \& Lada (1983), Wilking, Schwartz, \& Blackwell (1987), Wilking, Lada, \& Young (1989), Bouvier \& Appenzeller (1991), and from the Herbig \& Bell (1988) catalog (hereafter, HBC). We identified the objects having lunar occultations at observatories with suitable infrared equipment. The observable events occurred on five dates in 1991/1992; we succeeded in obtaining data on four of them (Table 1). For completeness, we include the occultation observations of 10 objects observed in 1986 and reported previously (Simon et al. 1987).

Table 2 lists the 35 systems observed by lunar occultation and imaging. According to the criteria of the works from which these systems were selected, all are believed to belong to the Ophiuchus SFR except IRS $32 \mathrm{~b}$ and IRS 56 for which definitive information is lacking (B. Wilking, private communication). Columns (1)-(4) list the object name; HBC number, if available; and position. The T Tauri star (TT) or weak-line T Tauri star (WT) nature of the object, using the $\mathrm{HBC}$ definition as to whether the $\mathrm{H} \alpha$ equivalent width is greater or less than $10 \AA$, is given in column (5). Column (6) provides the reference for the position and WT/TT type. Columns (7) and (8) list the total $K$ magnitude, $K_{\text {tot }}$, and specify when it was measured. We used $K_{\text {tot }}$ to apportion component fluxes of the binaries discovered by lunar occultation. Columns

TABLE 1

\begin{tabular}{lll}
\multicolumn{3}{c}{ Journal OF OPHIUCHUS OBSERVATIONS } \\
\hline \multicolumn{1}{c}{ Date } & Technique & Location \\
\hline 1. 1986, Jan 7 & Occultation & IRTF \\
2. 1991, Aug 19 & Occultation & AAT \\
3. 1992, Feb 25 & Occultation & IRTF \\
4. 1992, Jun 13 & Occultation & La Palma \\
5. 1992, Jul 11 & Occultation & Palomar \\
6. 1993, Apr 24-26 & Imaging & IRTF \\
\hline
\end{tabular}

(9) and (10) list $K-L$ colors and their references. For objects found to be multiple, the TT or WT designation, $K_{\text {tot }}$, and $K-L$ color, apply, in most cases, to the entry regarded as a single unresolved object. An $\mathrm{N}$ in column (9) indicates that Appendix A provides $(K-L)$ colors of the resolved components. We will use the WT/TT type and $K-L$ color in $\S 5$ as indicators of inner circumstellar disks and note that their measurement was not in general contemporaneous with that of $K_{\text {tot }}$. Column (11) summarizes the systems' multiplicity: S (single), B (binary), $\mathrm{T}$ (triple), and $\mathrm{Q}$ (quadruple) indicate the number of companions in the angular separation range 0 ."005-10" centered on the target. Column (12) identifies the occultation observations using the designations of Table 1. Tables 3 and 4 provide details of the singles and multiples and are described in $\S 2.3$. The multiplicity description is probably incomplete because subsequent observations may reveal additional companions and some of the apparent companions may not be bound. We discuss these issues in $\S 4.4$.

Seven of the 35 systems are in common with Ghez et al.'s (1993) $K$-band speckle imaging survey which included 24 objects in the Ophiuchus-Scorpius association. Our object list differs from theirs in three respects. The 35 systems are in the Ophiuchus SFR's dense central core or its vicinity; Ghez et al.'s objects are located over the greater area of the Oph-Sco association as well as in the core. Many of the 35 objects are IR-discovered, and only 14 have their TT/WT type determined. Ghez et al. selected objects with known TT/WT type. The limiting magnitude enters the two surveys in different ways. The Ghez et al. survey was limited to $K_{\text {tot }}=8.5 \mathrm{mag}$; our object list reaches somewhat fainter (Table 2). In practice the statistical analyses of both surveys are limited to companions with $K \leq 10$ mag.

The occultation data were obtained in the IR $K$ band by high-speed photometry with procedures described previously (Simon et al. 1992). The data were analyzed by fitting models and by inversion techniques (Chen et al. 1990 and Richichi et al. 1994).

\subsection{IR Imaging Observations}

Companions can be identified by the occultation technique out to $\sim 1^{\prime \prime}$ angular separation (Simon et al. 1992 and Fig. 4a). To search for companions at greater separation, we imaged all the objects successfully observed by occultation using ProtoCam, the near-IR camera of the NASA IRTF, in the IR $K$-band. It is equipped with a $58 \times 62$ pixel InSb array and permits observations at several angular scales. We imaged the objects in Table 2, except those in the area covered by Greene \& Young (1992), with pixel scale 0.35 pixel $^{-1}$ to a sensitivity of at least $K=12 \mathrm{mag}$. Greene \& Young's survey was carried out with a scale of $1^{\prime \prime} .2$ pixel $^{-1}$, so we used it to search for companions between $3^{\prime \prime}$ and $10^{\prime \prime}$ from the occultation-observed primary. We also imaged the objects in common with the Greene et al. survey using a 0 ". 135 pixel $^{-1}$ scale to a sensitivity of at least $K=12 \mathrm{mag}$ to search for closer companions that the occultation technique might have missed. No new companions were found.

In the occultation and imaging survey of the Taurus SFR (Simon et al. 1992), 10" was used as the radial upper bound out to which a pair of stars was considered a binary because at that radial separation contamination by field stars is negligible. The same radial limit can be applied to the Ophiuchus SFR. Greene \& Young (1992) measured a surface density of field stars brighter than $K=12$ mag of $\sim 5 \times 10^{-5}{\text { stars } \operatorname{arcsec}^{-2}}^{-2}$ 
TABLE 2

SYSTEMS OBSERVED IN OPHIUCHUS SFR

\begin{tabular}{|c|c|c|c|c|c|c|c|c|c|c|c|}
\hline Name & HBC & $\mathrm{RA}(1950)$ & $\overline{\operatorname{Dec}(1950)}$ & Type & $\begin{array}{l}\text { Ref } \\
\text { Posn } \\
\text { Type }\end{array}$ & $\begin{array}{l}\mathrm{K}_{t o t} \\
\mathrm{mag}\end{array}$ & $\begin{array}{c}\mathrm{Ref} \\
\mathrm{K}_{\text {tot }}\end{array}$ & $\begin{array}{l}\mathrm{K}-\mathrm{L} \\
\mathrm{mag}\end{array}$ & $\begin{array}{l}\text { Ref } \\
\text { K-L }\end{array}$ & Mult & $\begin{array}{l}\text { Occ } \\
\text { Obs }\end{array}$ \\
\hline ROXs 3 & 636 & 162247.6 & -244443 & WT & 1,4 & 8.8 & 2 & 0.18 & $\mathbf{A}$ & $\mathrm{S}$ & 2 \\
\hline SR 4 & 259 & 162254.87 & -241402 & TT & 1 & 7.4 & 6 & 0.5 & A & $\mathrm{S}$ & 5 \\
\hline DoAr 21 & 637 & 162301.67 & -241650 & WT & 1,4 & 6.3 & 6 & $0.4 \mathrm{~N}$ & B & $\mathrm{S}$ & 5 \\
\hline SR 3 & & 162307.7 & -242726 & & 2 & 6.5 & 6 & 0.52 & A & $\mathrm{S}$ & 1 \\
\hline GSS 29 & & 162315.5 & -241538 & & 2 & 8.2 & 6 & & & $\mathrm{~S}$ & 5 \\
\hline DoAr 24E & 639 & 162322.0 & -241414 & WT & 1,4 & 6.5 & 6 & $1.5 \mathrm{~N}$ & A & B & 5 \\
\hline GSS 32 & & 162322.6 & -241804 & & 2 & 7.7 & 6 & & & $\mathrm{~S}$ & 4 \\
\hline $\mathrm{S} 1$ & & 162332.8 & -241644 & & 2,4 & 6.3 & 6 & 0.47 & $\mathrm{E}$ & B & 5 \\
\hline SR $24 \mathrm{~N}^{*}$ & 262 & 162356.58 & -243854 & TT & 1 & 8.1 & 2 & 1.0 & $\mathrm{R}$ & $\mathrm{T}$ & 2 \\
\hline $\mathrm{S}^{*}$ & 262 & & & $\mathrm{TT}$ & 1 & 7.9 & 2 & 1.3 & $\mathrm{R}$ & & 2 \\
\hline WL 16 & & 162400.3 & -243044 & & 2 & 7.7 & 1 & 2.0 & WL & $S$ & 1 \\
\hline ELIAS 29 & & 162407.8 & -243033 & & 2 & 6.8 & 1 & 2.9 & $\mathrm{E}$ & $\mathrm{S}$ & 1 \\
\hline ELIAS 30 & & 162408.9 & $-24 \quad 1231$ & & 2 & 6.6 & 6 & 0.76 & $\mathrm{E}$ & B & 4 \\
\hline IRS $32 \mathrm{~b}$ & & 162411.8 & -243649 & & 2 & 10.0 & 6 & 1.2 & $\mathbf{A}$ & $S$ & 2,5 \\
\hline SR 12 & 263 & 162417.68 & -243500 & WT & 1,4 & 8.6 & 6 & 0.24 & W & B & 1,2 \\
\hline IRS 42 & & 162419.3 & -243503 & & 2 & 8.4 & 1 & 2.5 & W & $S$ & 1 \\
\hline YLW 15A & & 162424.9 & -243409 & & 2 & 9.9 & 2 & 3.1 & W & B & 2 \\
\hline YLW 16A & & 162426.0 & -243252 & & 2 & 8.8 & 1 & 3.2 & $\mathrm{~W}$ & $S$ & 1 \\
\hline IRS 48 & & 162435.5 & -242355 & & 2 & 7.6 & 3 & 1.5 & W & $\mathrm{S}$ & 3 \\
\hline IRS 49 & & 162436.4 & -243018 & & 2 & 8.4 & 1 & 1.2 & W & $\mathrm{S}$ & 1 \\
\hline $\mathrm{H} \alpha 52$ & & 162437.5 & -243236 & & 3 & 8.2 & 3 & 1.2 & A & $\mathrm{S}$ & 3 \\
\hline IRS 51 & & 162437.6 & -243635 & & 2 & 9.0 & 2 & 2.8 & $\mathrm{~W}$ & $\mathrm{~S}$ & 1,2 \\
\hline VSSG 14 & & 162448.3 & -241902 & & 2 & 7.3 & 6 & 0.54 & $\mathrm{E}$ & B & 4 \\
\hline IRS 56 & & 162449.0 & -244142 & & $2, \mathrm{~N}$ & 8.9 & 2 & & & $S$ & 1,2 \\
\hline ROXs 31 & 642 & 162450.1 & -243411 & WT & 1,4 & 8.2 & 2 & 0.43 & W & B & 1,2 \\
\hline SR 10 & 265 & 162454.05 & -241938 & $\mathrm{TT}$ & 1 & 8.8 & 6 & 0.90 & $\mathrm{R}$ & $\mathrm{S}$ & 4,5 \\
\hline $\mathrm{H} \alpha 60$ & & 162514.6 & -243021 & & 3 & 9.5 & 3 & 0.79 & $\mathrm{~A}$ & $\mathrm{~S}$ & 3 \\
\hline SR 20 & 643 & 162531.23 & -241608 & TT & 1,4 & 7.5 & 6 & 0.59 & $\mathrm{R}$ & $\mathrm{B}$ & 5 \\
\hline V853 Oph & 266 & 162543.67 & -242142 & $\mathrm{TT}$ & 1,4 & 8.0 & 6 & 0.54 & $\mathrm{R}$ & $\mathrm{T}$ & 5 \\
\hline $\mathrm{H} \alpha 63$ & & 162551.9 & $-2441 \quad 10$ & & 3 & 8.8 & 3 & 0.38 & A & $\mathrm{S}$ & 3 \\
\hline $\mathrm{H} \alpha 67$ & & 162721.0 & -244748 & & 3 & 9.6 & 3 & 0.64 & A & $\mathrm{S}$ & 3 \\
\hline ROXs 42B & & 162813.0 & -242619 & WT & 4 & 8.6 & 6 & & & B & 4,5 \\
\hline ROXs 43B & & 162818.2 & -242334 & WT & $4, \mathrm{~N}$ & 7.6 & 6 & 0.26 & WV & Q & 5 \\
\hline $\mathrm{H} \alpha 71$ & & 162829.2 & -241815 & & 3 & 8.4 & 6 & 2.3 & A & B & 5 \\
\hline Haro 1-16 & 268 & 162831.74 & -242110 & TT & 1,4 & 7.6 & 6 & 0.73 & $\mathrm{R}$ & $\mathrm{S}$ & 4,5 \\
\hline $\mathrm{H} \alpha 73$ & & 162851.7 & -245727 & & 3 & 10.0 & 3 & 1.1 & $\mathrm{~A}$ & $\mathrm{~S}$ & 3 \\
\hline
\end{tabular}

* Within $10^{\prime \prime}$, so form a system as defined in text.

REFERENCES.-Position and Type: (1) HBC; (2) Wilking et al. 1989; (3) Wilking et al. 1987; (4) Bouvier \& Appenzeller 1991. $K_{\text {tot }}$ : Numbering specifies observations in Table $1 . K-L:(\mathrm{A})$ this work, observation during period 6 in Table 1; (B) Bouvier \& Appenzeller 1991; (E) Elias 1978a; (R) Rydgren et al. 1976; (WL) Wilking \& Lada 1983; (W) Wilking et al. 1989; (WV) Walter et al. 1994.

in the vicinity of the Ophiuchus SFR. The probability of finding a field star in an area of $10^{\prime \prime}$ radius is therefore 0.017 . Taken together, the 35 areas of $10^{\prime \prime}$ radius areas centered on the systems in our survey might contain 0.6 field stars; they actually include 17 companions. Contamination by field stars is therefore insignificant within the $10^{\prime \prime}$ radius areas and we adopt the same $10^{\prime \prime}$ upper bound as for the Taurus occultation and imaging survey.

\subsection{Results: The Ophiuchus Entire Sample (ES)}

Table 3 lists the objects without detected companions in the angular separation range $0.005-10^{\prime \prime}$. Column (2) lists the position angle of the direction along which the Moon occulted the source, measured eastward from north. If more than one observation is available, all values are given because multiple observations can ensure that a companion was not missed as a result of a particular occultation direction. The system's $K$ magnitude, measured as close in time to the occultation as possible, appears in column (3). The limiting magnitude $K_{\text {lim }}$ in column (4) specifies the faintest companion that could have been detected by the occultation observation at separation $\geq 0$ ".020 on either side of the target. The value 0.020 was chosen because the sensitivity of the occultation survey to companions decreases at smaller separations (see $\S 4.3$ ). The $K_{\text {lim }}$ attained in an observation is determined by the dynamic range available, the sky brightness, and its stability (see Ridgway et al. 1977 for a thorough discussion of the limits of the occultation technique). $K_{\text {lim }}$ therefore varies from observation to observation. After analyzing the occultation data to determine the presence or absence of multiple components and the occultation rate (which sets the angular scale for a given observation), we returned to the data to estimate $K_{\text {lim }}$ by eye on the basis of experience. We used the faintest limit if several observations were obtained. Column (5) designates membership in the statistically complete subsamples we define in $\S 4.3$. Column (6) indicates the objects that were observed by Ghez et al. (1993) and Richichi et al. (1994).

Table 4 summarizes the parameters of the multiples in the systems observed. It includes all the companions of which we are aware; some were discovered in earlier observations (e.g., DoAr 24E by Chelli et al. 1988 and SR 20 by Ghez et al. 1993). The component $K$ magnitudes and separation, in milli- 
TABLE 3

OPHIUCHUS SFR SySTEMS WITH No DETECTED COMPANIONS IN SEPARATION RANGE $0.005-10^{\prime \prime}$

\begin{tabular}{llrrll}
\hline \hline Name & PA(deg) & $\mathrm{K}$ & $\mathrm{K}_{\text {lim }}$ & Subsample & Ref. \\
\hline & & & & & \\
ROXs 3 & 67 & 8.8 & 10.7 & FL & \\
SR 4 & 80 & 7.4 & 9.9 & K2 & 1 \\
DoAr21 & 95 & 6.3 & 9.7 & K2 & 1 \\
SR 3 & 138 & 6.5 & 9.3 & K2 & \\
GSS 29 & 69 & 8.2 & 9.9 & & \\
GSS 32 & 117 & 7.7 & 9.2 & & 2 \\
WL 16 & 140 & 7.7 & 10.2 & FL, K2 & \\
ELIAS 29 & 149 & 6.8 & 9.6 & K2 & \\
IRS 32b & 43,154 & 10.0 & 10.7 & FL & \\
IRS 42 & 132 & 8.4 & 10.0 & FL & \\
YLW16A & 145 & 8.8 & 9.8 & & \\
IRS 48 & 130 & 7.6 & 9.8 & K2 & \\
IRS 49 & 162 & 8.4 & 10.2 & FL & \\
H $\alpha 52$ & 105 & 8.2 & 10.4 & FL, K2 & \\
IRS 51 & 135,43 & 9.0 & 10.5 & FL & \\
IRS 56 & 137,79 & 8.9 & 10.9 & FL, K2 & \\
SR 10 & 103,60 & 8.8 & 11.1 & FL, K2 & 2 \\
H $\alpha 60$ & 123 & 9.5 & 10.5 & FL & \\
H $\alpha 63$ & 93 & 8.8 & 10.3 & FL & \\
H $\alpha 67$ & 91 & 9.6 & 10.8 & FL & \\
Haro 1-16 & 75,73 & 7.6 & 11.2 & FL, K2 & 1,2 \\
H $\alpha 73$ & 73 & 10.0 & 10.8 & FL & \\
\hline
\end{tabular}

REFERENCES.-(1) Ghez et al. 1993; (2) Richichi et al. 1994.

arcseconds (mas), appear in columns (2), (3), and (4). Column (5) gives the position angle from the brighter component to the fainter one measured eastward from north. Errors $(1 \sigma)$ are quoted only when they exceed $10 \%$ of the listed value. A single occultation measures only the projection of the actual separation along the occultation direction. Speckle, direct imaging, or multiple occultation observations can provide the actual values on the sky. Column (6) indicates whether the values in columns (4) and (5) are projected (P) or actual (A) values, and column (7) provides their source. Column (8) lists $K_{\text {Iim }}$, defined as for the singles. Column (9) indicates membership in the subsamples defined in $\S 4.3$. Column (10) indicates a note in Appendix A.

The triples are hierarchical in the sense that the ratio of the greatest separation to the smallest separation is large; it is greater than 10 for all the systems discussed here. The position angle for the triples is measured eastward from north of the brighter component to the fainter. The brighter component of a triple may be a single (e.g., SR 24S) or a close double (e.g., V853 OphA). The quadruple ROXs $43 \mathrm{~A} / \mathrm{B}$ is hierarchical in the same sense.

\section{THE TAURUS MULTIPLICITY SURVEY: NEW RESULTS}

The Taurus occultation and imaging survey currently includes 47 systems of which 19 are new occultation observations since the last comprehensive report (Simon et al. 1992). The target list and techniques of occultation and imaging observations are exactly the same as described previously. $K$-band images to supplement the occultation results were obtained with the facility cameras at the IRTF and Calar Alto Observatories. Table 5 lists the new occultation and imaging observations. Objects observed for the first time by occultation are listed in Roman font. Repeat observations of objects previously reported are indicated in italics (Tables 7 and 8 provide references). When possible, multiple observations were used together to obtain the actual orientation of the system on the sky. The La Palma and Calar Alto observations are described in detail by Richichi et al. (1994).

The 47 systems surveyed by occultation and imaging are listed in Table 6. Table 7 lists the systems without detected companions in essentially the same format as Table 3; column (7) indicates objects that were also observed in the Ghez et al. (1993) and Leinert et al. (1993) surveys and by Richichi et al. (1994). Table 8 presents the parameters of the multiples in our Taurus sample, again in the same format as Table 4 . There is much greater overlap with earlier work than there was for the

TABLE 4

Ophiuchus SFR Systems with Detected Companions in RANGe 0"005-10"

\begin{tabular}{|c|c|c|c|c|c|c|c|c|c|}
\hline Name & $\begin{array}{r}\mathrm{K}_{A} \\
\mathrm{mag}\end{array}$ & $\begin{array}{r}\mathrm{K}_{B} \\
\mathrm{mag} \\
\end{array}$ & $\begin{array}{l}\text { Sep. } \\
\text { mas }\end{array}$ & $\begin{array}{l}\text { PA } \\
\text { deg }\end{array}$ & $\begin{array}{l}\text { Proj/ } \\
\text { Act }\end{array}$ & $\begin{array}{l}\text { Ref } \\
\mathrm{P} \text { or A }\end{array}$ & $\begin{array}{r}\mathrm{K}_{l i m} \\
\mathrm{mag}\end{array}$ & Subsample & Notes \\
\hline \multicolumn{10}{|c|}{ Binaries } \\
\hline DoAr $24 \mathrm{E}$ & 6.9 & 7.9 & 2060 & 151 & A & 1,2 & 9.8 & K2 & \\
\hline $\mathrm{S} 1$ & 6.5 & 8.3 & 20 & 110 & $\mathrm{P}$ & 7 & 9.5 & $\mathrm{~K} 2$ & \\
\hline ELIAS 30 & 6.6 & 10.4 & 6700 & 175 & A & 0 & 9.4 & $\mathrm{~K} 2 \mathrm{~s}$ & \\
\hline SR 12 & 9.3 & 9.4 & $300 \pm 30$ & 85 & A & 5 & 10.5 & FL & \\
\hline YLW 15A & 9.9 & 12.7 & 7600 & 21 & A & 0,6 & 10.8 & FLs & $\mathrm{N}$ \\
\hline VSSG 14 & 7.8 & 8.6 & 101 & 89 & $\mathrm{P}$ & 7 & 10.0 & FL & \\
\hline ROXs 31 & 8.9 & 9.2 & $480 \pm 50$ & 262 & A & 0 & 11.2 & FL, K2 & $\mathrm{N}$ \\
\hline SR 20 & 7.6 & 9.8 & 71 & 225 & A & 2 & 10.5 & $\mathrm{FL}, \mathrm{K} 2 \mathrm{~s}$ & \\
\hline ROXs $42 \mathrm{~B}$ & 9.4 & 9.7 & 56 & 89 & $\mathbf{P}$ & 0 & 11.0 & FL & $\mathrm{N}$ \\
\hline $\mathrm{H} \alpha 71$ & 8.4 & 10.1 & 4800 & 37 & A & 0 & 11.0 & FLs, K2 & \\
\hline \multicolumn{10}{|c|}{ Triples } \\
\hline SR 24S & 7.9 & & 6000 & 60 & $\mathrm{~A}$ & 3 & 11.0 & $\mathrm{FL}, \mathrm{K} 2$ & \\
\hline $\mathrm{N}$ & 8.3 & 10.0 & 197 & 84 & A & 4 & & FL, K2 & \\
\hline V853 OphA & 8.9 & 9.1 & $13 \pm 2$ & 96 & $\mathbf{P}$ & 0 & 11.0 & & \\
\hline B & 9.9 & & 430 & 97 & A & 2 & & FLb, K2b & \\
\hline \multicolumn{10}{|c|}{ Quadruple } \\
\hline ROXs $43 \mathrm{~A}$ & 6.7 & & 4800 & 7 & A & 0,8 & & FLb, K2b & $N$ \\
\hline $43 \mathrm{~B}$ & 8.2 & 8.5 & $16 \pm 2$ & 89 & $\mathrm{P}$ & 0 & 10.5 & & \\
\hline
\end{tabular}

REFERENCES FOR ProjeCted or ACTUAL VALUES.-(0) This work; (1) Chelli et al. 1988; (2) Ghez et al. 1993; (3) HBC; (4) A. M. Ghez, private communication; (5) Simon et al. 1987; (6) Greene \& Young 1992; (7) Richichi et al. 1994; (8) Walter et al. 1994. 
TABLE 5

\begin{tabular}{|c|c|c|c|c|}
\hline & Date & Objects Observed & Location & Ref. \\
\hline 1. & 1990, Oct 6,7 & Imaging & IRTF & 1 \\
\hline 2. & 1991, Aug 5 & Haro 6-10, ZZ Tau & WIRO & 1 \\
\hline 3. & 1991, Sep 28-9 & $\begin{array}{l}\text { Haro } 6-10, \text { V927 Tau, HK Tau, V928 Tau } \\
\text { FY Tau, IW Tau, AA Tau }\end{array}$ & La Palma & 2 \\
\hline 4. & 1991, Sep 29 & V836 Tau & IRTF & 1 \\
\hline 5. & 1991, Oct 26 & GI Tau, $A A$ Tau, DN Tau, and Imaging & KPNO, IRTF & 1 \\
\hline 6. & 1992, Mar 11 & $\begin{array}{l}\text { FX Tau, V927, FZ Tau, El 12, GI Tau } \\
\text { DN Tau, CK St } 3 \text {, HD } 283759\end{array}$ & IRTF & 1 \\
\hline 7. & 1993, Jan 5 & $\begin{array}{l}\text { JH 108, HO Tau, FF Tau, HQ Tau, HP Tau } \\
\text { HP Tau/G3, HP Tau/G2, Haro 6-28, VY Tau }\end{array}$ & Calar Alto & 2 \\
\hline 8. & 1993, Feb 2 & HP Tau/G3, Haro 6-28, HQ Tau & IRTF & 1 \\
\hline 9. & 1993, Oct 2 & Imaging & Calar Alto & 1,2 \\
\hline
\end{tabular}

REFERENCES.-(1) This work; (2) Richichi et al. 1994.

Ophiuchus sample. Table 8 includes all the companions of the program objects of which we are aware. The companions of FV Tau/c, Haro 6-10, and IS Tau lie in the angular separation range that they could have been discovered or studied by the occultation technique but are fainter than the sensitivity limits of their occultation observation. They were discovered by imaging and speckle techniques and are included for completeness. As in Ophiuchus, all the triples are hierarchical. Appendix $B$ provides remarks for specific objects.

Leinert et al. (1993) surveyed the Taurus SFR for multiplicity at $K$ by speckle imaging. Their list contains 104 HBC objects brighter than $K=9.5 \mathrm{mag}$, including 37 of the systems in the present survey. Ghez et al. (1993) also included Taurus in their survey. They observed $45 \mathrm{HBC}$ objects, all with known TT/WT type, brighter than $K=8.5 \mathrm{mag} ; 18$ systems in the present survey are included in the Ghez et al. survey. Ten of the 47 systems in the present survey do not appear in either the Ghez et al. or Leinert et al. surveys.

\section{MULTIPLICITY IN THE TAURUS AND OPHIUCHUS SFRS}

\subsection{Introduction}

In this section we consider whether the star formation in Ophiuchus and Taurus produce multiples at the same frequency and with the same distribution of separations. We also compare the binary frequency among the young stars with that of the main-sequence solar-like stars.

Most of the Taurus objects in our sample, regarded as unresolved systems, can be placed in the H-R diagram (e.g., Strom et al. 1989). Their locations in the H-R diagram relative to pre-main-sequence evolutionary tracks indicate that most have masses in the range $0.5-1 M_{\odot}$ although the evolutionary tracks are imprecisely known (Hartigan et al. 1994 provide an instructive comparison). Only a few of the Ophiuchus objects have known spectral types because many are strongly obscured. Of the objects that can be located in the H-R diagram, $\sim 70 \%$ have masses in the range $0.5-1.5 M_{\odot}$ (e.g., Bouvier \& Appenzeller 1991).

Figure 1 shows the distributions of the total $K$ magnitude, $K_{\text {tot }}$, as given in Tables 2 and 6, of the objects in our Ophiuchus and Taurus samples. The medians are 8.2 and $8.0 \mathrm{mag}$ for the Ophiuchus and Taurus samples, respectively. The difference is insignificant for observing the objects by either occultation or imaging. We note, however that, because of the large obscuration of the Ophiuchus core (Wilking 1989), some of the objects in Ophiuchus must be intrinsically more luminous and more massive than those in Taurus. It is known, for example, that the S1 system contains a B4 star (Bouvier \& Appenzeller 1991). The masses of the objects in the Ophiuchus sample are thus more uncertain and distributed over a greater range than those in the Taurus sample. Nonetheless, it appears reasonable to compare the binary populations in Ophiuchus and Taurus to each other and to that of the main-sequence solar-like stars.

The entire samples (ES) of systems observed in the Ophiuchus and Taurus SFRs include companions discovered by lunar occultation, speckle techniques, and direct imaging. The ES provide a lower bound on the total binary frequency and their sensitivity to companions is nonuniform. We describe the ES in $\S 4.2$. In $\S 4.3$, we define two different subsamples with uniform sensitivity to compare the binary frequencies in Ophiuchus and Taurus. We compare the binary frequency of the ES and the solar-like stars in $\S 4.4$.

\subsection{Properties of the Entire Samples (ES) in Ophiuchus and Taurus}

In Ophiuchus, in the 0".005-10" angular separation range, 22 of the 35 systems have no detected companions, 10 are binaries, two are triples, and one is a quadruple (Table 9). The observed ratios of singles: binaries: triples: quadruple are therefore $63: 28: 6: 3$. There are at least 1.49 stars per system. The overall multiplicity, defined as the ratio of the number of multiples to the total number of systems, is $0.37 \pm 0.10$. This

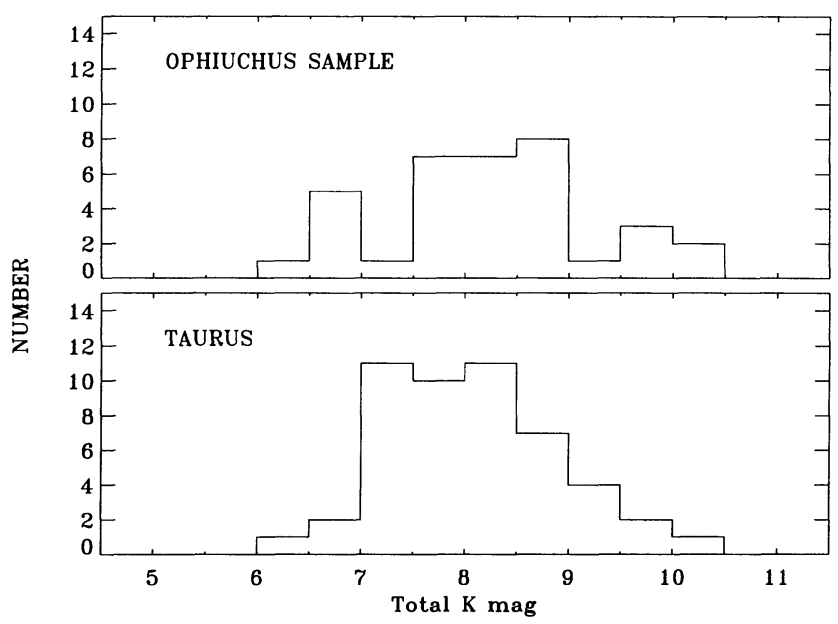

Fig. 1.-Distribution of total $K$-band magnitudes of the systems observed in Ophiuchus and Taurus (as listed in Tables 2 and 6 ). 
TABLE 6

SYSTEMS OBSERVED IN TAURUS SFR

\begin{tabular}{|c|c|c|c|c|c|c|c|c|c|c|c|}
\hline Name & HBC & RA(1950) & $\overline{\operatorname{Dec}(1950)}$ & Type & $\begin{array}{l}\text { Ref } \\
\text { Posn } \\
\text { Type }\end{array}$ & $\begin{array}{l}\mathrm{K}_{\text {tot }} \\
\mathrm{mag}\end{array}$ & $\begin{array}{c}\text { Ref } \\
\mathrm{K}_{\text {tot }}\end{array}$ & $\begin{array}{l}\mathrm{K}-\mathrm{L} \\
\mathrm{mag}\end{array}$ & $\begin{array}{l}\text { Ref } \\
\text { K-L }\end{array}$ & Mult & $\begin{array}{l}\text { Occ } \\
\text { Obs }\end{array}$ \\
\hline FS Tau & 383 & 41857.63 & 265030.50 & TT & 1 & 7.3 & $\mathrm{C} 1$ & 0.95 & $\mathrm{C}$ & B & $\mathrm{C} 1$ \\
\hline GU Tau & & 42335.19 & 26326.5 & & 2 & 9.0 & $\mathrm{~S} 2$ & 0.08 & A1 & S & $\mathrm{S} 2, \mathrm{~L}$ \\
\hline FV Tau & 386 & 42349.80 & 26013.0 & TT & 1 & 7.4 & $\mathrm{C} 1$ & $1: 21$ & A1 & B & $\mathrm{N}, \mathrm{C} 1, \mathrm{~L}$ \\
\hline FV Tau/c & 387 & 42350.73 & 2609.7 & TT & 1 & 8.7 & C1 & 0.93 & $\mathrm{~A} 1$ & B & $\mathrm{C} 1$ \\
\hline DF Tau & 36 & 42359.63 & 253541.7 & $\mathrm{TT}$ & 1 & 6.6 & $\mathrm{C} 1$ & 0.92 & $\mathrm{~K}$ & B & $\mathrm{C} 1$ \\
\hline DG Tau & 37 & 4241.01 & 255935.5 & TT & 1 & 6.8 & $\mathrm{C} 2$ & 1.70 & $\mathrm{~K}$ & $\mathrm{~S}$ & $\mathrm{~L}, \mathrm{C} 2$ \\
\hline Haro 6-10 & 389 & 42621.89 & 242630.0 & & 1 & 7.5 & A5 & 1.73 & $\mathrm{LH}$ & $\mathrm{B}$ & $\mathrm{N}, \mathrm{A}, \mathrm{R}$ \\
\hline FW Tau & & 42625.64 & 261022.0 & TT & 2,6 & 9.2 & $\mathrm{C} 1$ & 0.83 & A1 & $\mathrm{B}$ & $\mathrm{C} 1, \mathrm{~L}$ \\
\hline DH Tau & 38 & 42637.3 & 262628.9 & $\mathrm{TT}$ & 1 & 8.3 & $\mathrm{C} 1$ & 0.68 & $\mathrm{~K}$ & $\mathrm{~S}$ & $\mathrm{C} 1$ \\
\hline DI Tau & 39 & 42638.0 & 262620.1 & WT & 1 & 8.5 & $\mathrm{C} 1$ & 0.20 & $\mathrm{~K}$ & B & $\mathrm{C} 1$ \\
\hline IQ Tau & 41 & 42647.67 & 26016.3 & WT & 1 & 8.0 & $\mathrm{C} 1$ & 0.94 & $\mathrm{~K}$ & $\mathrm{~S}$ & $\mathrm{C} 1$ \\
\hline FX Tau & 44 & 42727.91 & 242018.2 & WT & 1,3 & 8.0 & A6 & 0.60 & $\mathrm{~K}$ & B & A \\
\hline DK Tau & 45 & 42740.48 & 255459.0 & $\mathrm{TT}$ & 1 & 7.0 & C1 & 1.30 & A1 & $\mathrm{B}$ & $\mathrm{N}, \mathrm{C} 1$ \\
\hline ZZ Tau & 46 & 42749.32 & 243556.9 & $\mathrm{TT}$ & 1 & 8.5 & A1 & 0.18 & $\mathrm{~K}$ & B & A \\
\hline V927 Tau & 47 & 42822.39 & 24429.7 & WT & 1 & 8.7 & A6 & 0.11 & $\mathrm{~K}$ & $\mathrm{~B}$ & $\mathrm{R}, \mathrm{A}$ \\
\hline HK Tau & 48 & 42848.85 & 241756.2 & $\mathrm{TT}$ & 1 & 8.4 & A5 & 0.74 & $\mathrm{~K}$ & B & $\mathrm{R}$ \\
\hline V928 Tau & 398 & 42917.16 & 24167.5 & WT & 1 & 7.8 & A1 & 0.1 & $\mathrm{~A} 1$ & B & $\mathrm{R}$ \\
\hline FY Tau & 401 & 42928.92 & 241338.6 & $\mathrm{TT}$ & 1 & 8.2 & A5 & 0.7 & A5 & $\mathrm{S}$ & $\mathrm{R}$ \\
\hline FZ Tau & 402 & 42930.08 & 241344.4 & $\mathrm{TT}$ & 1 & 7.2 & A6 & 1.42 & $\mathrm{E}$ & S & A \\
\hline UZ Tau $W^{*}$ & 53 & 42939.12 & 254613.4 & $\mathrm{TT}$ & 1 & 8.1 & S2 & 1.02 & $\mathrm{~A} 1$ & $\mathrm{~T}$ & $\mathrm{~S} 2$ \\
\hline $\mathrm{E}^{*}$ & 52 & 42939.40 & 254613.4 & $\mathrm{TT}$ & 1 & 7.3 & $\mathrm{~S} 2$ & 1.35 & A1 & & $\mathrm{S} 2, \mathrm{C} 1$ \\
\hline Elias 12 & 404 & 4305.2 & 24339 . & $\mathrm{TT}$ & 1 & 7.2 & A6 & 0.67 & $\mathrm{E}$ & $\mathrm{T}$ & A \\
\hline GI Tau & 56 & 43032.33 & 24153.1 & $\mathrm{TT}$ & 1 & 7.6 & A6 & 0.95 & $\mathrm{~K}$ & $S$ & A \\
\hline IS Tau & 59 & 43032.66 & 26334.5 & $\mathrm{TT}$ & 1 & 8.0 & $\mathrm{C} 1$ & 0.78 & $\mathrm{~K}$ & B & $\mathrm{C} 1$ \\
\hline GK Tau & 57 & 43032.76 & 241454.2 & $\mathrm{TT}$ & 1 & 7.4 & $\mathrm{~S} 1$ & 0.90 & $\mathrm{~K}$ & S & $\mathrm{S} 1$ \\
\hline DL Tau & 58 & 43036.02 & 251424.0 & TT & 1 & 8.0 & $\mathrm{~L}$ & 1.01 & $\mathrm{~K}$ & S & $\mathrm{L}$ \\
\hline IT Tau & & 43050.47 & 26714.1 & & 2 & 8.3 & $\mathrm{C} 1$ & 0.67 & $\mathrm{H}$ & B & $\mathrm{C} 1$ \\
\hline CI Tau & 61 & 43052.2 & 224416.7 & $\mathrm{TT}$ & 1 & 7.8 & $\mathrm{~S} 1$ & 0.93 & $\mathrm{~K}$ & S & $\mathrm{S} 1$ \\
\hline JH 108 & & 43111.14 & 224532.2 & WT & 4 & 9.5 & $\mathbf{H}$ & 0.20 & $\mathrm{H}$ & S & $\mathrm{R}$ \\
\hline AA Tau & 63 & 43153.45 & 242244.1 & $\mathrm{TT}$ & 1 & 7.8 & S1 & 1.03 & $\mathrm{~K}$ & S & $\mathrm{S} 1, \mathrm{~A}$ \\
\hline HO Tau & 64 & 43220.76 & 222606.6 & $\mathrm{TT}$ & 1 & 9.8 & $\mathrm{R}$ & 0.58 & $\mathrm{~K}$ & S & $\mathrm{R}$ \\
\hline FF Tau & 409 & 43220.92 & $2248 \quad 16.8$ & WT & 1 & 8.7 & $\mathrm{~S} 1$ & 0.43 & A1 & B & $\mathrm{S} 1, \mathrm{R}, \mathrm{A}$ \\
\hline DN Tau & 65 & 43225.68 & 24852.3 & $\mathrm{TT}$ & 1 & 8.0 & $\mathrm{~S} 1$ & 0.52 & $\mathrm{~K}$ & S & $\mathrm{S} 1, \mathrm{~A}$ \\
\hline CK St 3 & 411 & 43239.58 & 2451.8 & WT & 1,3 & 8.7 & A 6 & 1.2 & A5 & B & $\mathrm{N}, \mathrm{A}$ \\
\hline HQ Tau & & 43247.4 & 224416.1 & & 2 & 7.3 & $\mathrm{~S} 1$ & 1.35 & A1 & S & $\mathrm{N}, \mathrm{S} 1, \mathrm{R}, \mathrm{A}$ \\
\hline HP Tau & 66 & 43252.9 & $2248 \quad 17.7$ & TT & 1 & 7.6 & $\mathrm{~S} 1$ & 0.95 & K & B & $\mathrm{N}, \mathrm{S} 1, \mathrm{R}$ \\
\hline HP Tau/G3* & 414 & 43253.52 & 224804.0 & WT & 1 & 7.9 & A 8 & 0.07 & $\mathrm{~K}$ & $\mathrm{~T}$ & $\mathrm{R}, \mathrm{A}$ \\
\hline HP Tau/G2* & 415 & 43254.17 & 22488.2 & WT & 1,3 & 7.4 & S1 & 0.35 & $\mathrm{~K}$ & & $\mathrm{~S} 1, \mathrm{R}$ \\
\hline Haro 6-28 & 416 & 43256.81 & 224831.2 & $\mathrm{TT}$ & 1 & 9.3 & A 8 & 0.84 & $\mathrm{C}$ & B & $\mathrm{R}, \mathrm{A}$ \\
\hline HD 283759 & & 43347.29 & 24657.5 & & 2 & 8.3 & A6 & 0.0 & A6 & S & $A$ \\
\hline HT Tau & & 43446.75 & $26 \quad 18 \quad 0.9$ & & 2 & 7.2 & $\mathrm{~S} 2$ & 0.26 & $\mathrm{~A} 1$ & S & $\mathrm{S} 2$ \\
\hline DO Tau & 67 & 43524.18 & 26455.2 & $\mathrm{TT}$ & 1 & 7.3 & $\mathrm{~S} 2$ & 1.25 & $\mathrm{~K}$ & S & $\mathrm{S} 2$ \\
\hline HV Tau & 418 & 43530.9 & 26445.2 & WT & 1 & 7.8 & $\mathrm{~S} 2$ & 0.36 & A1 & $\mathrm{T}$ & $\mathrm{S} 2$ \\
\hline VY Tau & 68 & 43617.41 & $2242 \quad 2.3$ & $\mathrm{TT}$ & 1 & 9.0 & A6 & 0.47 & $\mathrm{~K}$ & B & $\mathrm{R}$ \\
\hline GN Tau & & 43617.04 & 253911.2 & $\mathrm{TT}$ & 2,6 & 8.4 & $\mathrm{~S} 2$ & 1.14 & $\mathrm{~A}$ & B & $\mathrm{S} 2$ \\
\hline Elias 18 & & 43651.8 & 253913.0 & & 5 & 6.3 & S2 & 1.76 & $\mathrm{E}$ & $\mathrm{S}$ & $\mathrm{S} 2$ \\
\hline Haro 6-32 & & 4380.0 & 255212.2 & & 2 & 10.0 & $\mathrm{~S} 2$ & 0.39 & A1 & $S$ & $\mathrm{~S} 2$ \\
\hline IW Tau & 420 & $438 \quad 1.94$ & 244521.8 & WT & 1 & 8.3 & A5 & 0.3 & A5 & B & $\mathrm{R}$ \\
\hline V836 & 429 & 5002.2 & 25196.9 & WT & 1 & 8.8 & A4 & 0.42 & K & $\mathrm{S}$ & $\mathrm{A}$ \\
\hline
\end{tabular}

* Within $10^{\prime \prime}$, so form a system as defined in text.

REFERENCES.-Position and Type: (1) HBC; (2) Jones \& Herbig 1979; (3) Strom et al.; (4) Hartmann et al. 1991 ; (5) Elias 1978b; (6) Briceno et al. 1993. $K_{\text {tot }}$ : (An) This work: $n$ specifies observation in Table 5 ; (C1) Chen et al. 1990; (C2) Chen et al. 1992; (L) Leinert et al. 1991; (S1) Simon et al. 1987; (S2) Simon et al. 1992. K - L: (An) This work, as for $K_{\text {to }}$; (C) Cohen \& Kuhi 1979; (E) Elias 1978b; (H) Hartmann et al. 1991; (K) Strom et al. 1989, and works cited therein; (LH) Leinert \& Haas 1989. Occultation Observations: (A) (see Table 5); (C1), (C2), (L), (S1), (S2) as for $K_{\text {tot }} ;(\mathrm{R})$ Richichi et al. 1994.

value is a lower bound because it does not include companions outside the angular limits of the survey, because the lunar occultation observations are less sensitive than the speckle and direct imaging observations, and because there are probably companions below the flux sensitivity limits of the three techniques. Figure 2 shows the observed distribution of separations of the Ophiuchus ES. Because the triples are hierarchical, we count each triple as contributing two separations. The smallest angular separation bin (0".0058-0".020) includes the angular resolution limit of the occultation technique and obviously is incomplete. The cutoff at $10^{\prime \prime}$ is the angular separation upper bound of our survey (see $\S 2.2$ ).

The Taurus ES of 47 systems includes 21 without detected companions, 22 binaries, and four triples (Table 9). The proportions of apparent singles: binaries: triples are $45: 47: 8$. The 47 systems therefore contain on average at least 1.64 stars per 
TABLE 7

TAURus SFR Systems With No DeteCted COMPANIONS IN SeParation Range 0".005-10"

\begin{tabular}{lllrrll}
\hline \hline Name & $\mathrm{N}_{\text {obs }}$ & PA(deg) & $\mathrm{K}$ & $\mathrm{K}_{\text {lim }}$ & Subsample & Ref. \\
\hline GU Tau & 2 & 104,107 & 9.0 & 10.7 & $\mathrm{FL}$ & 1,2 \\
DG Tau & 3 & & 6.8 & 9.7 & $\mathrm{~K} 2$ & $\mathrm{~N}, 1,2,3,4$ \\
DH Tau & 2 & 103,79 & 8.3 & 10.4 & $\mathrm{FL}, \mathrm{K} 2$ & 1,4 \\
IQ Tau & 3 & $120,54,122$ & 8.0 & 10.0 & $\mathrm{FL}, \mathrm{K} 2$ & $0,1,3,4$ \\
FY Tau & 1 & 37 & 8.2 & 10.1 & $\mathrm{FL}$ & $\mathrm{N}, 4,5$ \\
FZ Tau & 1 & 59 & 7.2 & 9.8 & $\mathrm{~K} 2$ & $0,3,4$ \\
GI Tau & 2 & 53,47 & 7.6 & 10.2 & $\mathrm{FL}, \mathrm{K} 2$ & $0,3,4$ \\
GK Tau & 1 & 92 & 7.4 & 8.8 & & $1,3,4$ \\
DL Tau & 1 & 78 & 8.1 & 9.8 & & 2,4 \\
CI Tau & 1 & 95 & 7.8 & 9.3 & & $1,3,4$ \\
JH 108 & 1 & 34 & 9.5 & 10.3 & FL & 5 \\
AA Tau & 3 & $92,39,71$ & 7.8 & 10.1 & FL,K2 & $1,4,5$ \\
HO Tau & 1 & 115 & 9.8 & 10.8 & FL & 5 \\
DN Tau & 2 & 12,64 & 8.0 & 10.4 & FL,K2 & $0,1,4$ \\
HQ Tau & 3 & $63,58,20$ & 7.4 & 9.8 & K2 & N,0,1,5 \\
HD 283759 & 1 & 63 & 8.2 & 10.7 & FL,K2 & 0 \\
HT Tau & 2 & 150,112 & 7.2 & 10.0 & FL,K2 & 1 \\
DO Tau & 1 & 66 & 7.3 & 9.8 & K2 & $1,3,4$ \\
Elias 18 & 1 & 48 & 6.3 & 9.3 & K2 & N,1 \\
Haro 6-32 & 1 & 73 & 10.0 & 10.8 & FL & N,1 \\
V836 & 1 & 110 & 8.8 & 11.0 & FL,K2 & 0,4 \\
\hline
\end{tabular}

REFERENCES.- (0) This work; (1) Simon et al. 1992; (2) Leinert et al. 1991; (3) Ghez et al. 1993; (4) Leinert et al. 1993; (5) Richichi et al. 1994. system. The overall multiplicity is $0.55 \pm 0.11$ which is again a lower bound. Figure 3 shows the distribution of separations in the Taurus sample.

Figure 4 shows the $K$ magnitude of the companions in the ES for Taurus and Ophiuchus versus their separation from the primary. The companions at separations $>1$ ", which are for the most part identified by imaging, include fainter stars than the companions detected by occultations, confirming the expectation that searching for companions by imaging is more sensitive than by occultation. Figure 4 also suggests a decrease in sensitivity to companions closer than $\sim 0^{\prime \prime} .010$, consistent with our experience that companions close to the angular resolution of the occultation technique are difficult to identify (Simon et al. 1992).

\subsection{Comparison of the Multiplicity in Ophiuchus and Taurus}

To compare the results for Ophiuchus and Taurus we define two subsamples of the data with uniform sensitivity to companions. Both are restricted to the range $0.020-10^{\prime \prime}$ to avoid the apparent roll-off in sensitivity at the closest separations (Fig. 4). The flux-limited sample (FL) includes systems satisfying the criteria $(a) K_{\text {lim }} \geq 10.0 \mathrm{mag}$, so that a companion as faint as $K=10$ could have been detected at separation greater than 0.020 from the primary; and $(b)$ if a companion

TABLE 8

Taurus SFR Systems with Detected Companions in Range 0".005-10"

\begin{tabular}{|c|c|c|c|c|c|c|c|c|c|}
\hline Name & $\begin{array}{c}\mathrm{K}_{A} \\
\mathrm{mag}\end{array}$ & $\begin{array}{r}\mathrm{K}_{B} \\
\mathrm{mag}\end{array}$ & $\begin{array}{l}\text { Sep. } \\
\text { mas }\end{array}$ & $\begin{array}{r}\mathrm{PA} \\
\mathrm{deg}\end{array}$ & $\begin{array}{l}\text { Proj/ } \\
\text { Act }\end{array}$ & $\begin{array}{l}\text { Ref } \\
\mathrm{P} \text { or } \mathrm{A}\end{array}$ & $\begin{array}{r}\mathrm{K}_{\text {lim }} \\
\mathrm{mag}\end{array}$ & Subsample & Notes \\
\hline \multicolumn{10}{|c|}{ Binaries } \\
\hline FS Tau & 7.4 & 9.7 & 265 & 60 & $\mathrm{~A}$ & 1 & 10.5 & $\mathrm{FL}, \mathrm{K} 2 \mathrm{~s}$ & \\
\hline FV Tau & 8.1 & 8.3 & 720 & 270 & A & $1,2,3,4$ & 9.9 & & \\
\hline $\mathrm{FV} \mathrm{Tau} / \mathrm{c}$ & 8.9 & 10.8 & 743 & 293 & A & $1,2,4$ & 9.9 & & \\
\hline DF Tau & 7.0 & 7.9 & 88 & 329 & A & 3 & 10.0 & $\mathrm{FL}, \mathrm{K} 2$ & \\
\hline Haro 6-10 & 7.6 & 9.8 & 1200 & 355 & A & 4,6 & 9.0 & & \\
\hline FW Tau & 10.0 & 10.0 & 160 & 160 & A & 1,2 & 10.6 & FL & \\
\hline DI Tau & 8.6 & 10.9 & 120 & 294 & A & 3 & 11.0 & FLs, K2s & \\
\hline FX Tau & 8.6 & 9.0 & 900 & 291 & A & $3,4,5$ & 10.0 & $\mathrm{FL}$ & \\
\hline DK Tau & 7.4 & 8.7 & 2530 & 115 & A & $1,3,4,5$ & 9.9 & $\mathrm{~K} 2$ & \\
\hline ZZ Tau & 8.9 & 9.8 & 29 & 244 & $\mathrm{P}$ & 5 & 9.8 & & \\
\hline V927 Tau & 9.3 & 9.6 & 300 & 290 & A & 4 & 10.0 & FL & \\
\hline HK Tau & 8.4 & 11.8 & 2400 & 175 & A & 8 & 10.1 & FLs & \\
\hline V928 Tau & 8.5 & 9.1 & 165 & 125 & A & 3,4 & 9.6 & & \\
\hline IS Tau & 8.0 & 10.6 & 221 & 92 & A & 3 & 9.3 & & $\mathrm{~N}$ \\
\hline IT Tau & 8.4 & 10.0 & 2480 & 225 & A & 1 & 10.1 & FL & \\
\hline FF Tau & 9.0 & 10.0 & 26 & 46 & $\mathrm{P}$ & 1,7 & 10.5 & FL & $\mathrm{N}$ \\
\hline CK St 3 & 8.9 & 10.8 & 2040 & 174 & A & 4,5 & 11.0 & FLs, K2 & \\
\hline HP Tau & 7.5 & 9.8 & 17 & 48 & $\mathrm{P}$ & 7 & 9.8 & & $\mathrm{~N}$ \\
\hline Haro 6-28 & 9.8 & 10.3 & 660 & 246 & A & 4 & 11.0 & FLs & \\
\hline VY Tau & 9.2 & 10.7 & 660 & 317 & A & 4 & 11.0 & FLs & \\
\hline GN Tau & 8.9 & 9.4 & 41 & 222 & $\mathrm{P}$ & 1 & 10.9 & $\mathrm{FL}, \mathrm{K} 2$ & \\
\hline IW Tau & 9.1 & 9.1 & 270 & 177 & A & 4 & 9.3 & & \\
\hline \multicolumn{10}{|c|}{ Triples } \\
\hline UZ Tau w & 8.4 & 9.5 & 340 & 0 & A & $1,3,4$ & 10.0 & $\mathrm{FL}$ & \\
\hline $\mathrm{e}$ & 7.3 & & 3780 & 273 & $\mathrm{~A}$ & $1,3,4$ & & $\mathrm{FL}, \mathrm{K} 2 \mathrm{~b}$ & \\
\hline Elias $12 \mathrm{~S}$ & 7.6 & & 410 & 330 & A & $3,4,5$ & 10.1 & FL, K2 & \\
\hline $\mathrm{N}$ & 9.1 & 9.1 & 23 & 95 & $\mathrm{P}$ & 5 & & $\mathrm{FL}, \mathrm{K} 2$ & \\
\hline HP Tau/G2 & 7.2 & & 9900 & 243 & A & $1,3,7$ & 9.8 & $\mathrm{~K} 2 \mathrm{~b}$ & \\
\hline G3 & 8.2 & 9.6 & 22 & 59 & $\mathrm{P}$ & 7 & 9.7 & & \\
\hline HV Tau & 8.3 & 8.9 & 35 & 273 & $\mathrm{P}$ & 1,4 & 10.0 & FLb & \\
\hline $\mathrm{C}$ & 11.6 & & 4000 & 45 & $\mathrm{~A}$ & 1 & & & \\
\hline
\end{tabular}

References for Projected or ACtual Values.-(1) Simon et al. 1992; (2) Leinert et al. 1991; (3) Ghez et al. 1993; (4) Leinert et al. 1993; (5) This work; (6) Leinert \& Haas 1989; (7) Richichi et al. 1994; (8) Moneti \& Zinnecker 1991. 
TABLE 9

Statistical Results

\begin{tabular}{|c|c|c|c|c|c|c|}
\hline \multirow[b]{2}{*}{ QUANTITY } & \multicolumn{2}{|c|}{$\begin{array}{c}\text { ENTIRE } \\
\text { SAMPLE (ES) }\end{array}$} & \multicolumn{2}{|c|}{$\begin{array}{l}\text { FLUX-LIMITED } \\
\text { SAMPLE (FL) }\end{array}$} & \multicolumn{2}{|c|}{$\begin{array}{c}\text { FLUX-RATIO- } \\
\text { LIMITED } \\
\text { SAMPLE (K2) }\end{array}$} \\
\hline & Tau & Oph & Tau & Oph & Tau & Oph \\
\hline Singles .... & 21 & 22 & 18 & 16 & 15 & 12 \\
\hline Binaries .................... & 22 & 10 & 9 & 7 & 6 & 6 \\
\hline Triples $\ldots \ldots \ldots \ldots \ldots \ldots \ldots \ldots$ & 4 & 2 & 2 & 1 & 1 & 1 \\
\hline Quadruples..$\ldots \ldots \ldots \ldots \ldots \ldots$ & 0 & 1 & 0 & 0 & 0 & 0 \\
\hline Totals $\ldots \ldots \ldots \ldots \ldots \ldots$ & 47 & 35 & 29 & 24 & 22 & 19 \\
\hline Stars/System .............. & 1.63 & 1.49 & 1.45 & 1.38 & 1.36 & 1.42 \\
\hline Singles . & 21 & 22 & 18 & 16 & 15 & 12 \\
\hline Multiples .................. & 26 & 13 & 11 & 8 & 7 & 7 \\
\hline$P\left(\chi^{2}\right)^{\mathrm{a}} \ldots \ldots \ldots \ldots \ldots \ldots \ldots \ldots$ & \multicolumn{2}{|c|}{0.11} & \multicolumn{2}{|c|}{0.73} & \multicolumn{2}{|c|}{0.73} \\
\hline Kolmogorov-Smirnov ${ }^{b} . . . \ldots$ & \multicolumn{2}{|c|}{0.33} & \multicolumn{2}{|c|}{0.61} & \multicolumn{2}{|c|}{0.93} \\
\hline
\end{tabular}

$\chi^{2}$ test applied to frequencies of singles and multiples.

b $\mathrm{K}-\mathrm{S}$ test applied to distribution of separations.

was detected, $K_{B} \leq 10.0$ mag. Definition of the FL subsample has the virtue of simplicity. Consider, however, searching for a companion of a pre-main-sequence star of specified age and mass in a system that belongs to the FL sample. The companion that may be present is presumed to have the same or younger age. If the primary is bright, either because it is relatively massive, young, or both, the range of secondary masses included within the $K_{\text {lim }}=10.0$ mag limit is greater than if the primary is faint, close to $K_{\text {lim }}$.

To avoid this possible problem we followed Ghez et al.'s approach (1993) and defined also a flux-ratio-limited subsample (K2). The singles and primaries in our sample are on the convective part of their pre-main-sequence evolutionary tracks (see Fig. 2 of Simon, Ghez, \& Leinert 1993) which are nearly vertical in the H-R diagram. The corresponding isochrones are approximately parallel. A sample of secondaries within a given fraction of the primary flux will span an approximately constant mass range regardless of age. Criteria for inclusion in the flux-ratio-limited sample (K2) are (a) $K_{\text {lim }}-K_{\text {tot }} \geq 2.0 \mathrm{mag}$ for singles, or $(b)$ if a companion was detected, $K_{\text {lim }}-K_{A} \geq 2.0$ and $K_{B}-K_{A} \leq 2.0$ mag. Members

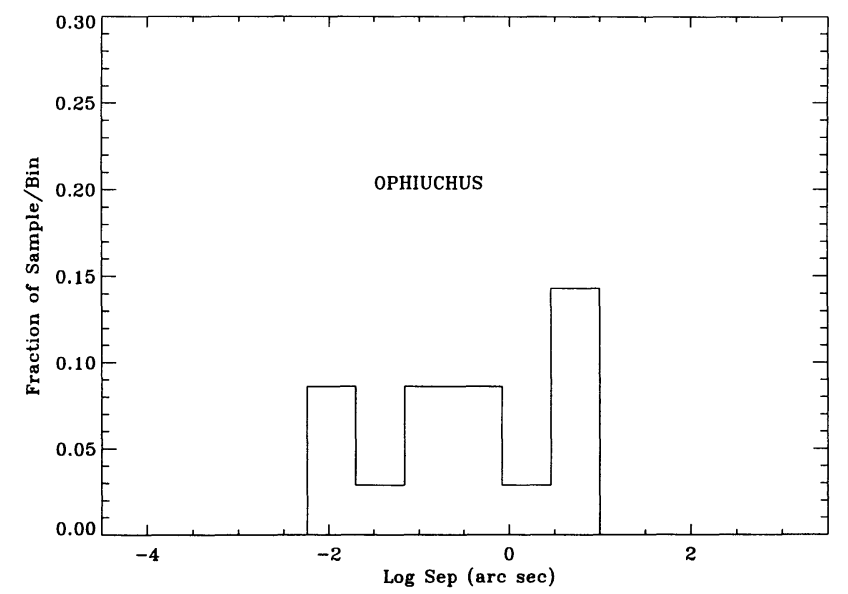

FIG. 2.-The observed distribution of separations in the Ophiuchus ES. The binning is $\Delta \log$ (separation arcseconds) $=0.540$, and the abscissa gives the fraction of the systems having separation in a given bin. Triples contribute two separations. of the K2 sample were observed with sufficient sensitivity to detect all companions within 2 mag of the primary in the angular separation range $00^{\prime \prime} 02-10^{\prime \prime}$.

Membership in the FL and $\mathrm{K} 2$ subsamples is indicated in column (5) of Tables 3 and 7, column (8) of Tables 4 and 8, and summarized in Table 9. The apparent multiple nature of an object in the FL or K2 may differ from that reported in the ES because a companion may be fainter or closer in separation than the subsample limits. ES binaries appearing as singles in the FL or K2 are designated FLs or K2s (e.g., YLW 15A in Ophiuchus), and ES triples appearing as binaries are designated FLb or K2b (e.g., V853A).

Are then the frequency of multiples and the distribution of separations the same in the Ophiuchus and Taurus SFRs? Are the stars in the Ophiuchus and Taurus FL or K2 subsamples drawn from the same "universal" population? We apply the $\chi^{2}$ test to the frequency of multiples and the KolmogorovSmirnov (K-S) test to the distribution of separations (Press et al. 1992). We binned the binaries and triples together because the triples' frequency is low (Siegel \& Castellan 1988). The $\chi^{2}$ test indicates that, for both the FL and K2 subsamples, $73 \%$ of independent trials of drawing subsamples from a universal young star population would yield a $\chi^{2}$ statistic greater than

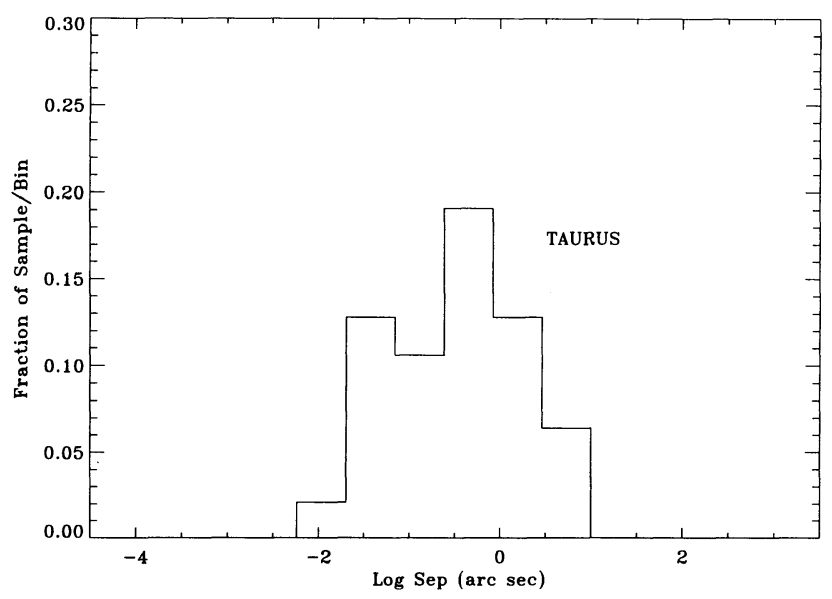

Fig. 3.-Same as Fig. 2 but for the Taurus survey 


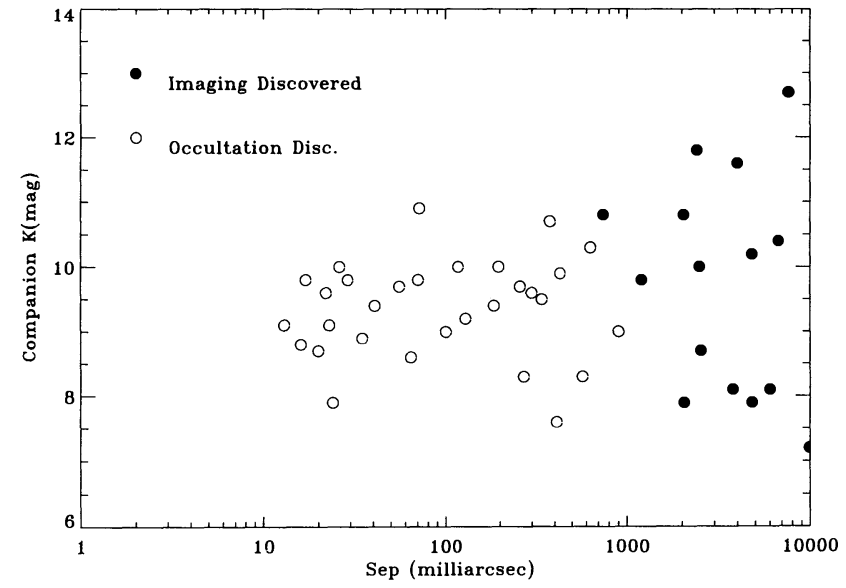

FIG. 4.- $K$-magnitude of the companion as a function of separation from the primary. For the occultation-discovered companions, the projected values of the separation, as observed, are used. The figure shows that the companions discovered by imaging tend to include fainter ones than those discovered by occultation and suggests a decrease in sensitivity to companions below about 20 mas separation.

the one we calculate (Table 9). The K-S test indicates that, for the FL and K2 subsamples respectively, $61 \%$ and $93 \%$ of repeated trials would yield a K-S statistic greater than the one actually calculated (Table 9). The FL and $\mathrm{K} 2$ subsamples of Ophiuchus and Taurus SFRs are therefore indistinguishable.

Moreover, application of the $\chi^{2}$ and K-S tests to the multiplicity and distribution of separations of the ES samples of Ophiuchus and Taurus, which look so different (Figs. 2 and 3, and summary in Table 9) yield the result that $11 \%$ and $33 \%$ of trials of drawing independent ES samples would have greater value of the $\chi^{2}$ and K-S measures than those actually obtained. We conclude that whatever the differences in our Ophiuchus and Taurus ES, their statistical significance is not very great.

\subsection{Comparison of the Entire Samples (ES) in Ophiuchus and Taurus with Main-Sequence Solar-like Stars}

We compare our results for Ophiuchus and Taurus, as given by the ES, with the study of Duquennoy \& Mayor (1991, hereafter DM) of the multiplicity among the nearby $G$ spectral type main-sequence stars. They measured the ratios of single: double: triple: quadruple systems as $57: 38: 4: 1$ in the orbital period range $-1<\log P$ (days) $<10$. They estimate that with correction for undetected orbits, the multiples probably represent close to $57 \%$ of their sample. DM fitted the distribution of orbital periods with a Gaussian peaking at $\log P($ days $)=4.8$ and standard deviation $\log P($ days $)=2.3$.

Our survey does not provide orbital periods. To compare the distributions we placed our measurements on an orbital period scale by assuming that the binaries were composed of two $0.5 M_{\odot}$ stars in circular orbits and that, on average, the measured angular separation corresponds to the semimajor axis. We did not correct the observed separations for orbital projection because the average correction is small for randomly oriented orbits (Fischer \& Marcy 1992, eq. [7]). It amounts to less than one-fifth of the binning interval we use to present our results (e.g., Figs. 2, 3, or 5). We also did not correct for the projection of the occultation observation because it does not change the distribution of separations significantly at separations greater than $\sim 0$ ". 01 . There are no binaries with projected separations $<0$ ".01 in our samples. For this comparison, we rebinned our data to the interval used by DM, $\Delta \log$ $P($ days $)=1$.

Figure 5 compares the distributions of separations in the Ophiuchus and Taurus ES with that of the solar-like stars. Over the range $0.020-10^{\prime \prime}$ angular separation, corresponding to 3-1400 AU linear separation, the average binary frequencies are $1.1 \pm 0.3$ and $1.6 \pm 0.3$ times that of the DM sample for the Ophiuchus and Taurus ES, respectively. These values are not corrected for incompleteness and are therefore lower bounds. The uncertainties include only $N^{1 / 2}$ counting statistics of the ES.

Figure 5 shows an apparent inconsistency. We found in $\S 4.3$ that the Ophiuchus and Taurus ES, and the FL and K2 subsamples are indistinguishable. Here we see that, while the binary frequency of the Taurus ES in the 3-1400 AU range is elevated over that of the solar-like stars (and consistent with previous findings, discussed below), the lower bound on the binary frequency of our Ophiuchus ES cannot be distinguished from that of the field stars. A numerical experiment suggests the explanation. We formed a modified Taurus ES with similar multiplicity as the field stars by halving the number of multiples and adding the other half to the singles, thereby keeping the total number of objects the same. Applying the $\chi^{2}$ test to compare the frequencies of the singles and multiples in the modified Taurus ES and the original Ophiuchus ES, we obtain that $36 \%$ of independent trials of drawing these samples from a universal sample would produce a $\chi^{2}$ value greater than the one we calculated. Evidently, these two samples are not very different, either. We conclude that, unfortunately our comparisons are limited by small sample size.

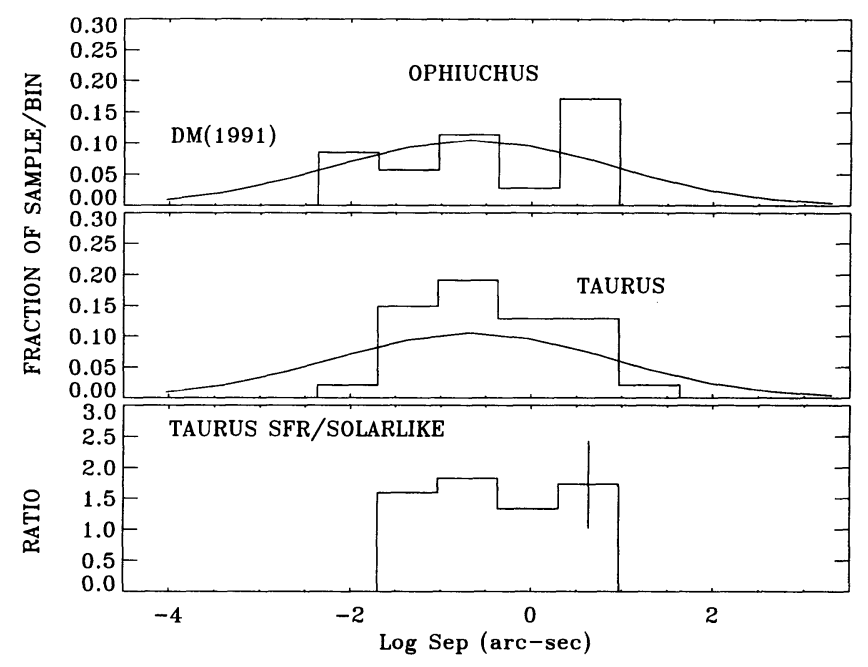

\begin{tabular}{|c|c|c|c|c|}
\hline 0 & 2 & $\begin{array}{lr}4 & 6 \\
\log P(\text { days })\end{array}$ & 8 & 10 \\
\hline
\end{tabular}

FIG. 5.-Top: The Ophiuchus ES (histogram) compared with the binary period distributions of the solar-like stars (smooth curve) measured by Duquennoy \& Mayor (1991). Conversion of observed separations of the Ophiuchus ES to orbital periods assumes two $0.5 M_{\odot}$ stars in circular orbits at $140 \mathrm{pc}$ distance (see text). The histogram bin size is $\Delta \log P($ days $)=1$, the same as DM's. On a $\Delta \log$ (separation arcseconds) basis, the bin size is 0.667 , somewhat larger than used in Figs. 2 and 3. Middle: Same as the top panel, but for the Taurus ES compared to the solar-like stars. Bottom: From the middle panel, the ratio of the binary frequency per bin in the Taurus ES to the solar-like binaries. Representative $\pm 1 \sigma$ errors are shown. The average ratio over the four bins, corresponding to $3-1400 \mathrm{AU}$ separation, is $1.6 \pm 0.3$. 
Leinert et al. (1993) found that in the angular separation range $0.13-13^{\prime \prime}(18-1800 \mathrm{AU})$ the binary frequency in Taurus is $1.9 \pm 0.3$ times greater than DM's value for the solar-like stars. This value is also derived directly from the observations without correction for incompleteness so it is also a lower bound. For the Taurus-Auriga and Ophiuchus-Scorpius SFRs taken together, Ghez et al. (1993) determined an excess binary frequency of $3.5 \pm 1.2$ in the angular separation range 0 ".11-1".8 (16-252 AU). This value contains a correction for incompleteness; the observed value of the ratio, before correction, derived by binning the observed binaries as here is $2.3 \pm 0.5$. Taken together, the present results, and the Ghez et al. and Leinert et al. surveys, agree in finding an excess binary frequency in Taurus relative to the solar-like stars of at least a factor of $\sim 2$ over the separation range 3-1800 AU.

In the Oph-Sco SFR alone, Ghez et al. identified nine binaries out of 24 systems surveyed in the angular separation range $0.1-1 " .8$, their range of completeness (16-252 AU at Oph-Sco). This corresponds to an observed binary frequency of $2.4 \pm 0.6$ times that of the solar-like stars. The overlap of the Ghez et al. survey and the Ophiuchus ES includes seven objects, of which one is a binary in this separation range. The binary frequency of Ghez et al. objects not observed in common $(8 / 17)$ is $2.9 \pm 1.0$ that of the solar-like stars. It is interesting that the Ophiuchus ES and Ghez et al. samples can be so different.

\section{PHYSICAL PROPERTIES OF THE SINGLES AND MULTIPLES}

\subsection{Multiplicity and Circumstellar Disks}

The observational evidence is strong that low-mass young stars are surrounded by geometrically thin but optically thick (in the visible and near-IR) disks that can be quite extensive (Beckwith 1994). The observations suggest a distinction between inner and outer disk regions. The inner disks, extending to a few photospheric radii, are responsible for hydrogen line and near-IR continuum excess emission (Basri \& Bertout 1989). The outer disks, identified by the long-wavelength continuum emission of their dust and spectral line emission of their molecular gas, may extend to several hundred $\mathrm{AU}$ (Beckwith et al. 1990). The samples of systems we observed in Ophiuchus and Taurus are dominated by objects with prominent $\mathrm{H} \alpha$ spectral line emission and IR excesses, simply because they have drawn attention and therefore represent the majority of young stars that have been identified. On the other hand, the $\mathrm{X}$-ray-selected young stars, which do not have prominent circumstellar emission, appear to outnumber the classically iden- tified young stars by a factor of $2-5$ (Walter et al. 1994). Could the large binary star frequency in Ophiuchus and Taurus apply mostly to the classically selected systems? In other words, could it be the consequence of a selection effect?

Further, theoretical calculations and observations show that tidal interactions in binary systems can force the evolution of the associated circumstellar disks and alter their structure (Artymowicz \& Lubow 1994; Dutrey et al. 1994; Ghez et al. 1994). Our current understanding is that planets form by rapid accumulation of planetesimals in the circumstellar disk associated with the birth of a star (Lissauer 1993). Are the disks, and hence environments for planet formation, in single and multiple systems different?

We investigate first whether the presence of an inner disk affects the observed multiplicity. We used two indicators for inner disks, one the TT/WT distinction, and the other the $(K-L)$ color. Kenyon \& Hartmann (1990) and Edwards, Ray, $\&$ Mundt (1993a) have shows that a $(K-L)$ greater than 0.30.5 mag indicates optically thick inner disks and correlates with spectral line emission associated with $\mathrm{T}$ Tauri activity. We adopt $K-L \geq 0.4$ mag of the combined system as the discriminant for the presence of an optically thick inner disk.

We merged the Ophiuchus and Taurus samples to consider the physical properties because they should not depend on the multiplicity of the SFR. Table 10 summarizes the total multiplicity, defined as the ratio of the number of binaries, triples, and quadruples to the total number of systems, by WT and TT type and by $(K-L)$ color $<0.4$ and $\geq 0.4 \mathrm{mag}$. Within the uncertainties, the multiplicity appears to be independent of the presence of an inner disk. The results of the merged FL and K2 subsamples are consistent with these, but since the number of objects in each category is smaller, the corresponding estimated errors are larger. Leinert et al. (1993) and Ghez et al. (1993) also found no difference in the multiplicity of the WTs and TTs.

Among the multiples, do the systems with and without inner disks have different distributions of binary separations? We used the WT/TT and $(K-L)$ color criteria again. For the WT/TT criterion, we merged the Ophiuchus and Taurus FL and K2 subsamples, separated the WTs and TTs and show the binary frequency for each as a function of separation in Figure 6. There is no statistically significant difference in the WT and TT distributions over the range of separations 3-1400 AU $\left(0.02-10^{\prime \prime}\right)$. For the comparison using the $(K-L)$ color, we divided the merged Ophiuchus and Taurus ES into the $(K-L)<0.4$ and $(K-L) \geq 0.4$ mag groups. Figure 6 shows that there is no clear cut difference between the two. Ghez et al.

TABLE 10

Multiplicity AND INNER Disks

\begin{tabular}{|c|c|c|c|}
\hline \multirow[b]{2}{*}{ Multiplicity } & \multirow{2}{*}{$\begin{array}{c}\text { DM (1991) } \\
\text { RESULTS }\end{array}$} & \multicolumn{2}{|c|}{ Merged Ophiuchus and TaURUS } \\
\hline & & ES & Inner Disk Criterion \\
\hline$\frac{B+T+Q}{S+B+T+Q} \ldots \ldots$ & $0.49-0.57$ & $\frac{39}{82}=0.48 \pm 0.08$ & $\begin{array}{r}\text { WT: } \frac{14}{19}=0.74 \pm 0.19 \\
\text { TT: } \frac{18}{33}=0.55 \pm 0.12 \\
K-L<0.4: \frac{9}{16}=0.56 \pm 0.19 \\
K-L \geq 0.4: \frac{29}{62}=0.47 \pm 0.08\end{array}$ \\
\hline
\end{tabular}




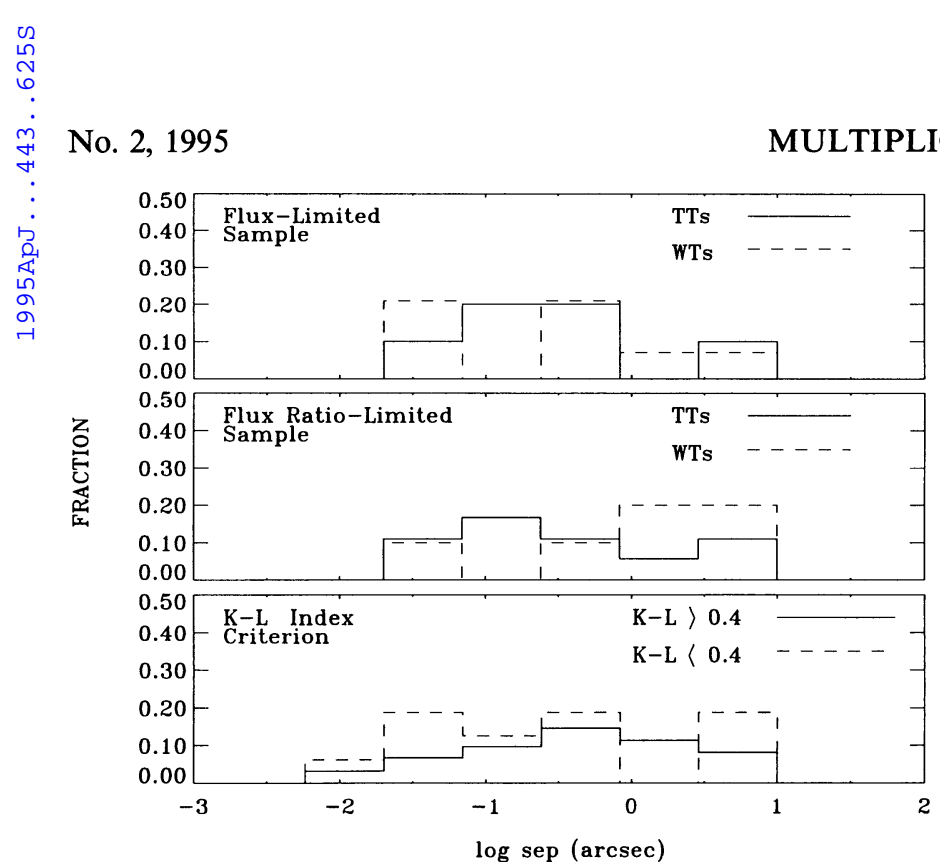

FIG. 6.-The binary frequency as a function of separation using the WT/TT and $K-L$ color as indicators of inner disks. The binning is the same as in Figs. 2 and 3. Top: The binary frequency distribution for the blended Ophiuchus and Taurus FL samples which together contain 14 WT systems (singles and multiples) and 20 TTs. Middle: Same as the top panel but for the blended K2 sample which contains 10 WTs and 18 TTs. Bottom: The binary frequency distribution of the blended Ophiuchus and Taurus ES samples using the $K-L$ discriminant. The blended ES samples contain a total of 16 systems with $K-L<0.4$ and 62 with $K-L \geq 0.4$.

(1993) found that in their sample the binary frequency of the WTs exceeded that of TTs at the closest separations (16-40 AU). This effect does not appear here, possibly because both our samples and Ghez et al.'s involve small numbers of binaries, so the statistical significance is low.

We used Beckwith et al.'s (1990) and André \& Montmerle's (1994) surveys of the $1.3 \mathrm{~mm}$ flux density of the objects in Taurus and Ophiuchus ES, respectively, to consider the presence of dusty outer disks in the systems with and without detected companions in our sample. These surveys included 30 systems in the merged ES without detected companions; 19 were detected at $1.3 \mathrm{~mm}$. The surveys included 29 systems in the merged ES with detected companions; 12 of these were detected. Figure 7 shows that the systems without detected companions are stronger $1.3 \mathrm{~mm}$ continuum sources (median $84 \mathrm{mJy}$ ) than the multiple systems (median $62 \mathrm{mJy}$ ), suggesting that disks are less massive in the multiple systems.

We are thus unable to find strong evidence of an effect of multiplicity on inner disks, as indicated by the WT/TT type or $K-L$ index, or that the presence of inner disks biases the measurement of the multiplicity. On the other hand, we do find that the multiples are weaker sources of millimeter wavelength continuum emission. Both findings are plausible. The inner disk indicators identify regions within a few photospheric radii, deep in the gravitational potential well of the associated star. Since the closest of the binaries in our sample are at projected separation $\sim 3 \mathrm{AU}$, their inner disks may not be strongly perturbed by the relatively distant companion. On the other hand, if the outer disks are to be detected by their millimeter-wave dust thermal continuum emission, they must be relatively massive $\left(\sim 0.01 M_{\odot}\right)$ and extensive $(\sim 100 \mathrm{AU})$ (Beckwith et al. 1990). The typical separation of the binaries in our samples is $\sim 50 \mathrm{AU}$; outer disks in such systems are therefore particularly vulnerable to tidal distortion and dissipation.

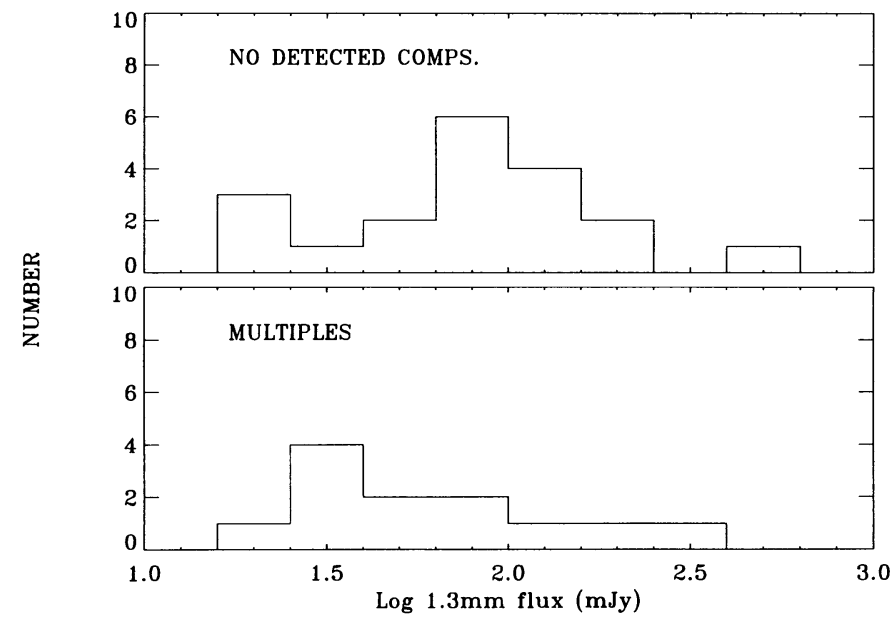

FIG. 7.-Distribution of $1.3 \mathrm{~mm}$ continuum flux density for the objects with and without detected companions. The Ophiuchus and Taurus samples are blended, and the $1.3 \mathrm{~mm}$ flux densities are from Beckwith et al. (1990) and André \& Montmerle (1994). Only objects with $1.3 \mathrm{~mm}$ detections are shown.

\subsection{Why Are There So Many Binaries?}

Stars are believed to form in dense molecular cloud cores (e.g., Benson \& Myers 1989). The physical parameters of these regions must determine the types and multiplicity of the stars that are formed. The angular momentum of the star-forming material is a critical parameter because its conservation during collapse can force rotational breakup of the protostar. Goodman et al. (1993) measured the rotation of a number of molecular cloud cores. We estimated the specific angular momentum of the binaries in Taurus by assuming two $0.5 M_{\odot}$ stars in circular orbits. Figure 8 shows that the distribution of the specific angular momentum of the cores in Taurus-Auriga overlaps that of the young star binaries in Taurus. (We did not merge the Ophiuchus binaries because the Goodman et al. sample includes only one molecular cloud core in Ophiuchus.) The overlap must be much greater than Figure 8 indicates. The $10^{\prime \prime}$ separation limit of our survey excludes the widely spaced binaries which have the greatest angular momenta. Low values

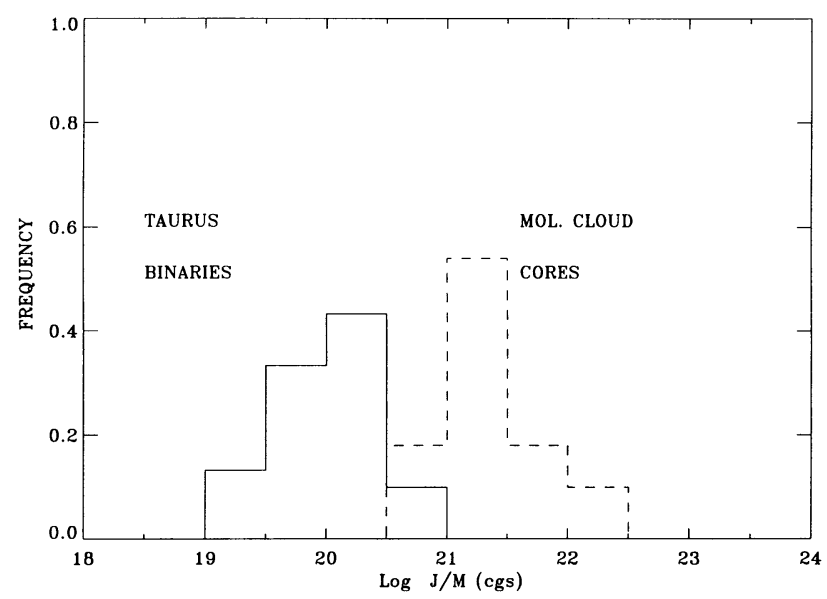

Fig. 8.-The specific angular momentum distribution of the young star binaries in Taurus compared with that of the known rotating molecular cloud cores in Taurus-Auriga (Goodman et al. 1993). The number of objects per bin is plotted along the abscissa. Both distributions are incomplete as discussed in the text. 
are probably underrepresented because the rotation of slowly rotating cores is difficult to measured. The significance of Figure 8 is simply that the young binary and molecular cloud distributions overlap. This is what we would expect if the angular momenta of the binaries originates in the rotation of the molecular cloud cores.

The typical orbital angular momentum of a binary, $10^{53}$ $10^{54} \mathrm{~g} \mathrm{~cm}^{2} \mathrm{~s}^{-1}$, is more than 10 times greater than that of the extensive circumstellar disks (Beckwith et al. 1990), and about 1000 times greater than the observed rotational momenta of the T Tauri stars (Hartmann et al. 1986; Bouvier et al. 1993; Edwards et al. 1993b). The orbital motion of the stars in a binary represents nearly all the angular momentum of the system. Figure 8 suggests that star-forming regions contain so many binaries because molecular cloud cores rotate and the collapsing protostars solve their angular momentum problem by forming binaries.

\section{CONCLUSIONS}

We have sampled 35 young star systems in the Ophiuchus SFR for companions by the combined techniques of lunar occultation and imaging observations in the $K$ band. To avoid significant contamination by field stars, we adopt $10^{\prime \prime}$ as the separation upper bound at which we consider a pair of stars as forming a gravitationally bound system. The 35 systems include at least 10 binaries, two triples, and one quadruple. In the range of projected separations 3-1400 AU, the binary frequency is at least $1.1 \pm 0.3$ that of the solar-like stars. These measures of companionship are lower bounds because we do not correct for incompleteness.

Our lunar occultation and imaging survey of the Taurus SFR now includes 47 systems; these include at least 22 binaries and four triples. The binary frequency in the Taurus SFR is at least $1.6 \pm 0.3$ that of the nearby solar-like stars.

Whether the binary frequencies and distribution of separations of the Ophiuchus and Taurus SFRs can be distinguished from each other and whether they differ from that of the field solar-like stars are critical questions for this work. The samples we observed in Ophiuchus and Taurus are indistinguishable, but this is not a very significant statement because while we find a binary frequency in Taurus greater than or equal to about twice that of the field stars, the binary frequency of the Ophiuchus sample is only at least that of the field. Unfortunately, the small sample size precludes more definitive comparisons.

Nonetheless, all the surveys of young star multiplicity that have been carried out, in diverse SFRs, find a binary frequency that is greater than or equal to that of the field stars in the range of separations to which they are sensitive (e.g., Mathieu 1994 for observations of spectroscopic binaries; the imaging surveys of Reipurth \& Zinnecker 1993 and Prosser et al. 1994; and the surveys of Ophiuchus and Taurus we have discussed). It appears that star formation produces mostly binaries.

We find no effect of the presence of inner ( $<1 \mathrm{AU}$ size) circumstellar disks on the observed multiplicity or the distribution of separations. On average, the singles in our sample are stronger $1.3 \mathrm{~mm}$ sources than the multiples suggesting that multiplicity does affect the outer $(\sim 100$ AU size $)$ disks. The specific angular momenta of the binaries overlap that of the molecular cloud cores. Binary formation may be common simply because molecular clouds rotate.

We thank the Time Allocation Committees and Directors of the AAT, Calar Alto, Kitt Peak, La Palma, NASA-IRTF, Palomar, and WIRO observatories for the time allocations that made this research possible. We thank the staffs of these observatories who supported us in making these observations. M. S. thanks Bruce Wilking and Bob Mathieu for scrutinizing Tables 2 and 6 and for comments on cluster membership; Rwei-Ju Chuang and Nancy Mendell for advice on statistics; and Lisa Prato for careful readings of early drafts. We thank Bob Mathieu, the referee, for urging us to sharpen the arguments in $\$ \$ 4.3$ and 4.4 and suggesting the numerical experiment. A. G.'s work is supported by NASA through grant HF 1031.92A awarded by the Space Telescope Science Institute which is operated by the Association of Universities for Research in Astronomy, Inc., for NASA under contract NAS 5-26555. Infrared astrophysics at Palomar is supported by a grant from the NSF. M. S.'s work is supported in part by NSF grant AST 91-4606.

\section{APPENDIX A}

\section{NOTES FOR OPHIUCHUS SFR OBJECTS}

DoAr 21.-Bouvier \& Appenzeller (1991) report $K-L$ variation 0.4-0.8 over 3 days.

DoAr 24E.-The component brighter at $K$ (Table 4) has $K-L=0.9$ and the fainter at $K$ has $K-L=2.3$.

YLW 15A.-Companion is GY 263 (Greene \& Young 1992).

IRS 56.- Insufficient information is available to be certain that it is an association member; the position listed in Wilking et al. (1989) is in error (B. Wilking, private communication 1993).

ROX 31.- The actual values are the solution obtained by combining the IRTF (1/86) and AAT (8/91) data. At projected separation $\sim 69 \mathrm{AU}$, the stars should not have moved a measurable amount in the $5 \mathrm{yr}$ interval between the observations.

ROXs 42B.--Result quoted is from data obtained at Palomar; it agrees with the La Palma result (Richichi et al. 1994).

ROXs 43A.- Occultation observations revealed that ROXs 43B is a close binary. ROXs $43 \mathrm{~A}, 6$, 2 away, is a spectroscopic binary (Mathieu, Walter, \& Myers 1989). We did not observe the lunar occultation of ROXs 43A; nonetheless, the ROXs 43A/B system is at least a quadruple. 


\section{APPENDIX B}

\section{NOTES FOR TAURUS SFR OBJECTS}

FV Tau.-The $K-L$ value in Table 6 is for the composite, unresolved system. For the eastern component $K-L=1.1$, and for the western, $K-L=1.2$.

FV/c Tau.-The companion was discovered by direct imaging (Simon et al. 1992); at $K=10.8$, it is fainter than the $K_{\text {lim }}$ of the occultation observation.

DG Tau.-Data along position angles $90^{\circ}, 233^{\circ}$, and $256^{\circ}$ show resolved emission (Leinert et al. 1991; Chen et al. 1992).

Haro 6-10.-The companion was discovered by speckle interferometry (Leinert \& Haas 1989); at $K=9.8$ it fell below the $K_{\text {lim }}$ of the subsequent occultation observation. The listed $K-L$ is for the combined unresolved system; for the northern component $K-L=3.16$, and for the southern, $K-L=1.23$ (Leinert \& Haas 1989).

DK Tau.-The listed $K-L$ is for the combined, unresolved system. Separately, the values are $K-L=1.41$ for the primary and 0.85 for the secondary.

FY Tau.--Richichi et al. (1994) consider it a suspected binary.

IS Tau.-The companion was discovered by speckle imaging (Ghez et al. 1993). At $K=10.6$, the companion is fainter than the $K_{\text {lim }}$ of the occultation observation.

FF Tau.-The values listed are from Richichi et al. (1994). We did not combine them with the earlier observation (Simon et al. 1987) to derive an actual separation on the sky because substantial orbital motion in the $8.5 \mathrm{yr}$ separating the observations is likely.

$C K$ St 3.-The $K-L$ listed is for the combined, unresolved system. For the northern component, $K-L=1.4$ and for the southern, $K-L=1.3$.

$H Q$ Tau.- Simon et al. (1987) reported it as a close binary. The best analysis by Chen et al. (1990) yields separation $=9$ mas, $\mathrm{PA}=63$, flux ratio 6.9. It appeared as an unresolved single object in occultation observations on 1993 January 5 (Richichi et al. 1994) and 1993 February 2 (this work); at the sensitivity of these observations, the companion should have been detected. Although it is possible that the companion eluded detection by its location along its orbit, it seems best to note that the original detection of a companion was at the limits of the occultation technique, to list HQ Tau as a single, and to regard it as an unconfirmed binary.

HP Tau. - Simon et al. (1987) reported it as unresolved along PA $=85^{\circ}$. The $K_{\text {lim }}$ for that observation was $9.7 \mathrm{mag}$. The companion identified by Richichi et al. (1994) at $K_{B}=9.8 \mathrm{mag}$ is therefore consistent with the earlier nondetection. It lies at the sensitivity limits of the technique.

Elias 18.--Either extended or binary (Simon et al. 1992).

Haro 6-32.-Either extended or binary (Simon et al. 1992).

André, P., \& Montmerle, T. 1994, ApJ, 420, 837

Artymowicz, P., \& Lubow, S. H. 1994, ApJ, 421, 651

Basri, G., \& Bertout, C. 1989, ApJ, 341, 340

Beckwith, S. V. W. 1994, in Theory of Accretion Disks-2, ed. W. J. Duschl et al. (Dordrecht: Kluwer), 1

Beckwith, S. V. W., Sargent, A. I., Chini, R. S., \& Güsten, R. 1990, AJ, 99, 924

Benson, P. J., \& Myers, P. C. 1989, ApJS, 71, 89

Bouvier, J., \& Appenzeller, I. 1991, A\&AS, 92, 481

Bouvier, J., Cabrit, S., Fernández, M., Martín, E. L., \& Matthews, J. M. 1993, A\&A, 272, 176

Briceno, C., Calvet, N., Gomez, M., Hartman, L. W., Kenyon, S. J., \& Whitney, B. A. 1993, PASP, 105, 686

Chen, W. P., Howell, R. R., Simon, M., \& Benson, J. A. 1992, ApJ, 387, L43

Chen, W. P., Simon, M., Longmore, A. J., Howell, R. R., \& Benson, J. A. 1990, ApJ, 357, 224

Chelli, A., Zinnecker, H., Carrasco, L., Cruz-Gonzáles, I., \& Perrier, C. 1988, A\&A, 207, 46

Cohen, M., \& Kuhi, L. V. 1979, ApJS, 41, 743

de Geus, E. J., \& Burton, W. B. 1991, A\&A, 246, 559

Duquennoy, A., \& Mayor, M. 1991, A\&A, 248, 485 (DM)

Dutrey, A., Guilloteau, S., \& Simon, M. 1994, A\&A, 286, 149

Edwards, S., Ray, T., \& Mundt, R. 1993a, in Protostars and Planets III, ed.

E. H. Levy \& J. I. Lunine (Tucson: Univ. of Arizona Press), 567

Edwards, S., et al. 1993b, AJ, 106, 372

Elias, J. H. 1978a, ApJ, 224, 453 .1978b, ApJ, 224, 857

Fischer, D. A., \& Marcy, G. W. 1992, ApJ, 396, 178

Ghez, A., Emerson, J. P., Graham, J. R., Meixner, M., \& Skinner, C. 1994, ApJ, 434,707

Ghez, A., Neugebauer, G., \& Matthews, K. 1993, AJ, 106, 2005

Goodman, A. A., Benson, P. J., Fuller, G. A., \& Myers, P. C. 1993, ApJ, 406, 528

Greene, T. P., \& Young, E. T. 1992, ApJ, 395, 516

Hartmann, L., Hewett, R., Stahler, S., \& Mathieu, R. D. 1986, ApJ, 309, 275

Hartmann, L., Jones, B. F., Stauffer, J. R., \& Kenyon, S. J. 1991, AJ, 101, 1050

Herbig, G., \& Bell, K. R. 1988, Lick. Obs. Bull., 1111 (HBC)

Hughes, J., Hartigan, P., Krautter, J., \& Kelemen, J. 1994, AJ, submitted

Jones, B. F., \& Herbig, G. H. 1979, AJ, 84, 1872

\section{REFERENCES}

Kenyon, S. J., \& Hartmann, L. W. 1990, ApJ, 349, 190

Kenyon, S. J., Hartmann, L. W., Strom, K. M., \& Strom, S. E. 1990, AJ, 99, 869 Leinert, Ch., \& Haas, M. 1989, ApJ, 342, L39

Leinert, Ch., Haas, M., Richichi, A., Zinnecker, H., \& Mundt, R. 1991, A\&A, 250, 407

Leinert, Ch., Zinnecker, H., Weitzel, N., Lenzen, R., Haas, M., Christou, J., Ridgway, S., \& Jameson, R. 1993, A\&A, 278, 129

Lissauer, J. J. 1993, ARA\&A, 31, 129

Mathieu, R. D. 1994, ARA\&A, 32, 465

Mathieu, R. D., Adams, F. C., \& Latham, D. W. 1991, AJ, 101, 2184

Mathieu, R. D., Walter, F. M., \& Myers, P. C. 1989, AJ, 98, 987

Moneti, A., \& Zinnecker, H. 1991, A\&A, 242, 428

Press, W. H., Flannery, B. R., Teukolsky, S. A., \& Vetterling, W. T. 1992, Numerical Recipes (Cambridge: Cambridge Univ. Press)

Prosser, C. F., Stauffer, J. R., Hartmann, L., Soderblom, D. R., Jones, B. F., Werner, M. W., \& McCaughrean, M. J. 1994, ApJ, 421, 517

Reipurth, B., \& Zinnecker, H. 1993, A\&A, 278, 81

Richichi, A., Leinert, Ch., Jameson, R., \& Zinnecker, H. 1994, A\&A, 287, 145

Ridgway, S. T., Wells D. C., \& Joyce, R. R. 1977, AJ, 82, 414

Rydgren, A. E., Strom, S. E., \& Strom, K. M. 1976, ApJS, 30, 307

Sargent, A. I., \& Beckwith, S. V. W. 1991, ApJ, 382, L31

Siegel, S., \& Castellan, N. J., Jr. 1988, Nonparametric Statistics for the Behavioral Sciences (New York: McGraw Hill)

Simon, M., Chen, W. P., Howell, R. R., Benson, J. A., \& Slowik, D. 1992, ApJ, 384,212

Simon, M., Ghez, A. M., \& Leinert, Ch. 1993, ApJ, 408, L33

Simon, M., \& Guilloteau, S. 1992, ApJ, 392, L47

Simon, M., Howell, R. R., Longmore, A. J., Wilking, B. A., Peterson, D. M., \& Chen, W. P. 1987, ApJ, 320, 344

Strom, K. M., Strom, S. E., Edwards, S., Cabrit, S., \& Skrutskie, M. F. 1989, AJ, 97, 1451

Walter, F. M., Vrba, F. J., Mathieu, R. D., Brown, A., \& Myers, P. C. 1994, AJ, 107,692

Wilking, B. A. 1989, PASP, 101, 229

Wilking, B. A., \& Lada, C. J. 1983, ApJ, 274, 698

Wilking, B. A., Lada, C. J., \& Young, E. T. 1989, ApJ, 340, 823

Wilking, B. A., Schwartz, R. D., \& Blackwell, J. H. 1987, AJ, 94, 106 